\title{
PARALLEL POLYMORPHISM IN THE HETEROCHROMATIN OF TRILLIUM SPECIES
}

\author{
C. D. DARLINGTON and G. W. SHAW \\ Botany School, Oxford
}

Received 3.vi.58

\section{INTRODUCTION}

THE mechanisms of heredity are similar in all organisms but the mechanisms of variation are widely different. The study of chromosome structure, especially of heterochromatin, reveals the differences and helps us to understand them.

The ten chromosomes of Trillium contracted at mitosis are a quarter of a millimetre long. They are also marked by larger segments of heterochromatin than any other plants. These $\mathrm{H}$-segments, to use Rutishauser's abbreviation, are groups of genes which differ from the rest in two respects. They remain condensed within the resting nucleus; and they are exactly recordable at metaphase following cold treatment. This is due to their deficiency in DNA which is shown by a weak Feulgen reaction and a narrower chromatid (Darlington and La Cour, I94I, Shaw, I958, I959, Heyes and Shaw, 1958).

Variations occur within the species, and indeed within populations, in the numbers, sizes and positions of $\mathrm{H}$-segments. The variations are discontinuous so that the species may be described as polymorphic in the sense of Ford. The range of variation within populations can then be compared among species. Homozygotes and heterozygotes in respect of every difference can be distinguished and counted. By this means the character of polymorphism in heterochromatin may be compared with that shown in other organisms at both the chromosome and the phenotypic levels.

\section{MATERIALS AND METHODS}

Ten species of Trillium and one species of Paris have been used (table $\mathrm{I}$ ). Their naming was confirmed with the help of Bailey (194I), Gray (1950) and Britton and Brown (1952).

It is important to know for our purpose how far these systematists' species are genuine units, genetically isolated from one another, not interbreeding in nature and therefore not overlapping in variation either at the chromosome or the phenotypic level. Our smaller collections do not enable us to be certain of the answer to this question. Our larger collections, however, indicate that $T$. luteum and $T$. sessile may be only colour polymorphisms within a genetic species governed by a supergene difference. The same kind of distinction may exist between $T$. erectum and T. cernuum.

Our genuine species may thus be less than ten. On the other hand we are confident that the three main sub-divisions into which we have divided the species in table ro are absolutely isolated: making the most conservative estimate, as we must in arriving at our conclusions, our plants are of three species. On a reasonable estimate they are eight. 
Geographical Distribution. The American species of Trillium are in two groups. The western species ( $T$. chloropetalum and $T$. ovatum) are confined to the coast states. The eastern species range from Nova Scotia and Minnesota to Florida and Arkansas. There appears to be more heterochromatin in those species with the more northerly range. T. stylosum with least heterochromatin has a limited distribution which is also the most southerly.

Nomenclature. Three plants named T. stylosum and five named T. grandiflorum (3-7) by Darlington and La Cour correspond with a pattern we now recognise (by the D) as belonging to $T$. recurvatum. Some plants named $T$. grandiflorum by P. C. Bailey (1954) on the other hand (by A and B) seem to be T. erectum. Bailey's single

TABLE I

\begin{tabular}{|c|c|c|c|c|c|}
\hline Species & & $\begin{array}{c}\text { Chromosome } \\
\text { no. }(x=5)\end{array}$ & $\begin{array}{l}\text { No. of } \\
\text { plants }\end{array}$ & Source & References \\
\hline 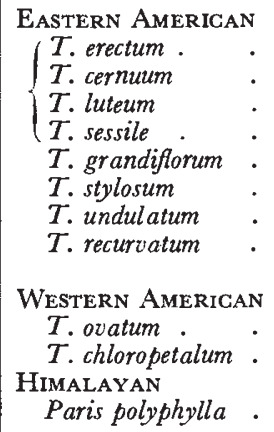 & 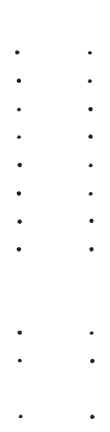 & $\begin{array}{l}2 x, 0-6 f f, 3^{x} \\
2 x, 0-2 f f \\
2 x, 0-2 f f \\
2 x, 0-3 f f \\
2 x, 0-2 f f \\
2 x \\
2 x \\
2 x \\
\\
2 x, f \\
2 x \\
2 x, 0-1 f\end{array}$ & $\begin{array}{r}70 \\
45 \\
30 \\
20 \\
65 \\
20 \\
10 \\
1\end{array}$ & $\begin{array}{c}\text { Messrs Perry } \\
,, \\
, " \\
, \\
, \\
\text { Oxford Bot. } \\
\text { Garden } \\
\text { Edinburgh* } \\
\text { Worcester* } \\
\text { Edinburgh* }\end{array}$ & $\begin{array}{c}2,3,4,8 \\
9 \\
5 \\
2,5 \\
2,6, \text { Io, I I } \\
\text { None } \\
3 \\
2 \\
\\
\text { None } \\
\text { None } \\
\\
\text { 1, } 7\end{array}$ \\
\hline
\end{tabular}

I. Darlington and La Cour, $193^{8 .}$

2. Darlington and La Cour, I940.

3. Wilson and Boothroyd, I94 I, I944.

4. P. C. Bailey, 1949.

5. P. C. Bailey, 1952.

6. P. C. Bailey, I954.
7. La Cour, I95I.

8. Boothroyd, I953.

9. Rutishauser and La Cour, 1956. ro. Giles and Wilson, 1956.

I I. Rutishauser, I956b.

* We are indebted to Captain R. G. Berkeley of Spechley Park, Worcester, and the Director of the Royal Botanic Garden, Edinburgh, for gifts of plants of these species. Perry's seven eastern species were collected by Mr E. C. Robbins of MacDowell Co., in the west of North Carolina, the common ground of these species.

plant named $T$. recurvatum with only a single $\mathrm{H}$-segment (terminal in B) does not correspond with any combination we have yet found in a single individual.

The five pairs of chromosomes of Trillium seem to correspond in all species and are labelled A, B, C, D and E (figs. I-8).* The European system which we shall follow has been to label the chromosomes $\mathrm{A}$ to $\mathrm{E}$ in descending order of size from $36 \mu$ to $22 \mu$. The American and Japanese equivalents we assume are given below :

$\begin{array}{llllll}\text { Eur } & \text { A } & \text { B } & \text { C } & \text { D } & \text { E } \\ \text { Jap } & \text { A } & \text { C } & \text { B } & \text { E } & \text { D } \\ \text { Am } & \text { E } & \text { B } & \text { D } & \text { C } & \text { A }\end{array}$

* In one instance only (table 6 , footnote), the distinction between $\mathrm{C}$ and $\mathrm{D}$ was uncertain and interchange could have taken place. Interchange in evolution is, however, effectively absent. This is not surprising since we know that in Paris there is proximal localisation of chiasmata such as would sterilise interchange heterozygotes (Darlington, I94 I). 
Supernumerary chromosomes $(f)$ are of two types: subterminals $\left(s^{1}, s^{2}\right)$, and telocentrics $\left(t^{0}, t^{1}, t^{2}\right)$.

In tables 2-9 each variant of a chromosome type has been given a number from I to I4. When a chromosome is homozygous, the number is given once only. When heterozygous, both numbers are given.

Where the mitotic complements of two or more plants in a species correspond exactly in their $\mathrm{H}$-segment pattern, and hence are cytologically indistinguishable, the plants are provisionally assumed to belong to a single clone. The analysis of the plants examined is given in terms of these clones in tables $2-9 .{ }^{*}$

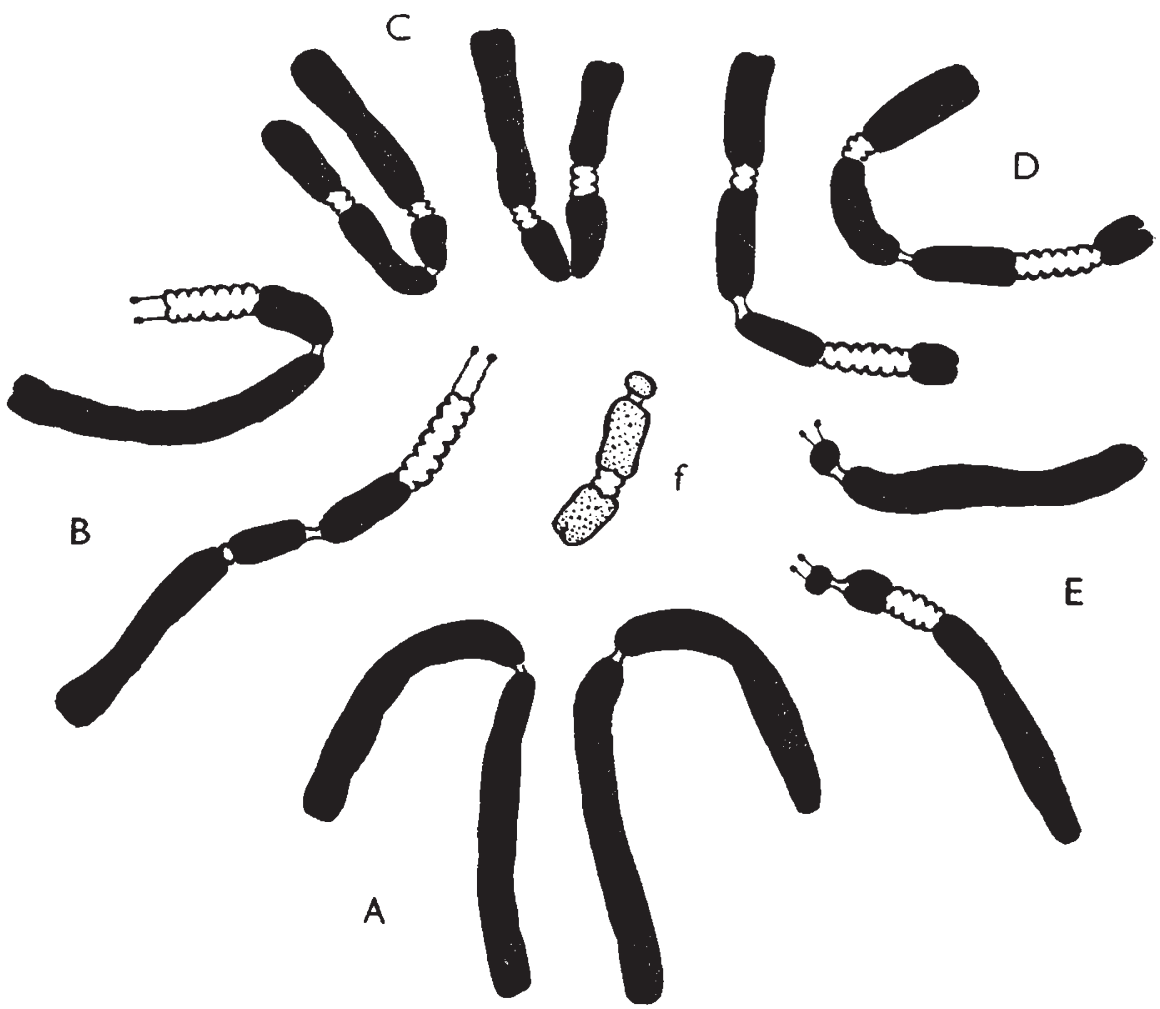

Fig. I.-Chromosome complement of $T$. ovatum from root showing twelve DNA-starved H-segments.

Formula : A1.1, B9.14, $\mathrm{C}_{7}, 7, \mathrm{D}_{5}{ }^{\prime} \cdot 5^{\prime}, \mathrm{E} 1.2, f^{h}$ or $s^{2}$.

Note.-B and $\mathrm{E}$ have nucleolar satellites; these are specific to their own loci in each species but are not recorded in our formulæ and diagrams since they are usually collapsed in other species. B and $\mathrm{E}$ happen to be the only heterozygous chromosomes in this complement. See figs. 6 and 10. $\times 1800$.

Techniques. Plants were kept at $\mathrm{r}^{\circ}-2^{\circ} \mathrm{C}$. for 96 hours before fixation. Where chromosomes showed signs of stickiness, a further 24 hours in colchicine $(0 \cdot 0$ r per cent.) at $r^{\circ}$ C. was given before fixation.

Root tips were taken from potted plants and fixed one hour in $2 \mathrm{BD}$, hydrolysed for $\mathrm{r} 6$ minutes at $6 \mathrm{o}^{\circ}$ C. in N.HCl, and stained one hour in Feulgen solution. Squash preparations were dehydrated in Cellosolve (Shaw, r950, r956), cleared in xylene and mounted in Canada balsam.

* We do not believe this assumption to be true since the multiple clones assumed on this rule are those having the commonest chromosome types. This is, how ever, the most modest assumption in relation to our arguments since it und er estimates heterozygosity. 


\section{CHROMOSOME COMPLEMENTS OF THE SPECIES}

I. Trillium erectum (28 clones). Two chromosome types new for the genus were found in this species. B 12 occurred in 19 of the 28 clones. E 7 was in a single triploid all of whose chromosomes were homozygous. The triploidy did not modify the differential reaction of the $\mathrm{H}$-segments.

The novel type of chromosome in the triploid, the first found among American populations, is an instance of the principle that polyploids, owing to their selective

FIGs. 2-6.-Diagrams of complements in ten species of Trillium levelled at the centromeres: showing all the types of each chromosome found in each species. $\times c .1200$.

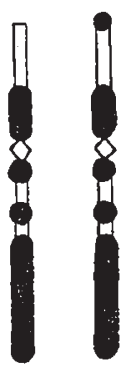

BII

$B / 2$
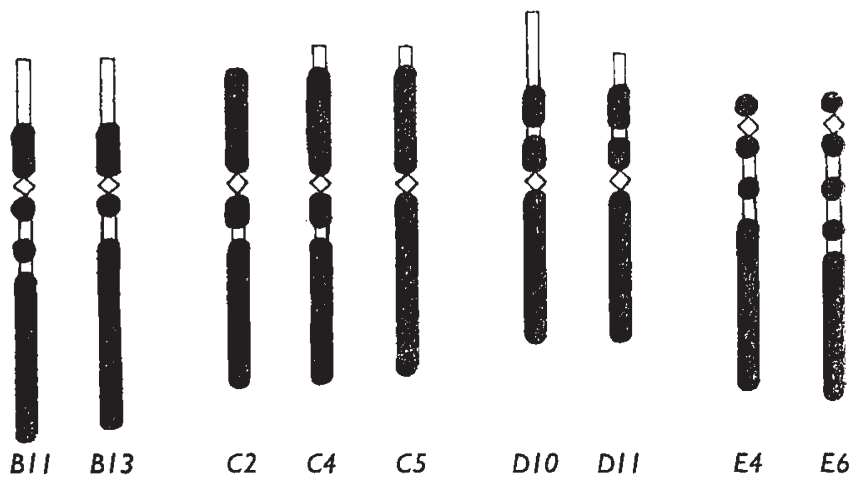

T. cernuum

Fig. 2

survival, are unrepresentative of the diploid populations from which they have arisen.

More H-segments are illustrated by Wilson and Boothroyd (1941, 1944) than we or other workers have found. Their photographs suggest artefacts.

I. T. kamtschaticum as described by Darlington and La Cour seems to correspond to our T. erectum.

2. T. cernuum ( 22 clones). Like $T$. erectum this species has a proximal $\mathrm{H}$-segment in each arm of $\mathrm{A}$ and in the long arm of B. These segments distinguish the two species from all the rest. Our plants contain more $\mathrm{H}$-segments than those of Rutishauser and La Cour (1956).

3. T. luteum ( 28 clones). The terminal $\mathrm{H}$-segment on $\mathrm{B}$ occurs in a series of lengths $(\mathrm{o}, \mathrm{I} \cdot 5 \mu, 3 \mu, 4 \cdot 5 \mu$ and $6 \mu)$. The intercalary $\mathrm{H}$-segment in $\mathrm{C}$ also occurs in two lengths.

Bailey's (1952) plant would appear to have the formula AI.B8.C4..D5. EI. (homozygous). 
4. T. sessile ( 13 clones). All the i i types of chromosome found in this species, except $C_{1}$, occur also in the larger sample of $T$. luteum.

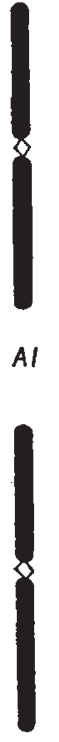

AI

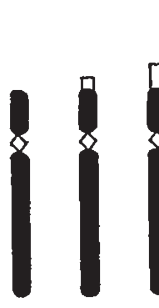

B6

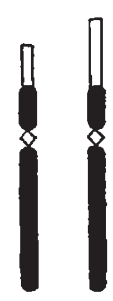

B9 B10

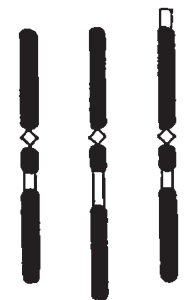

C2 $\quad$ C3 $\quad$ C4

T. luteum

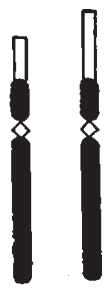

B9 $\mathrm{B} 10$

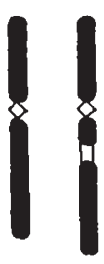

$\mathrm{Cl} \mathrm{C2}$

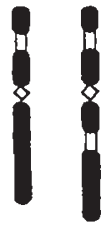

D4 D5

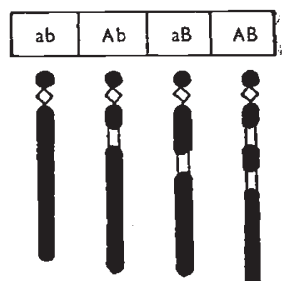

E1 E2 E3

E4

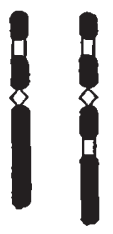

D4 D5

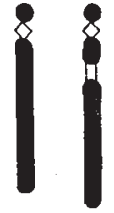

EI E2

Fig. 3
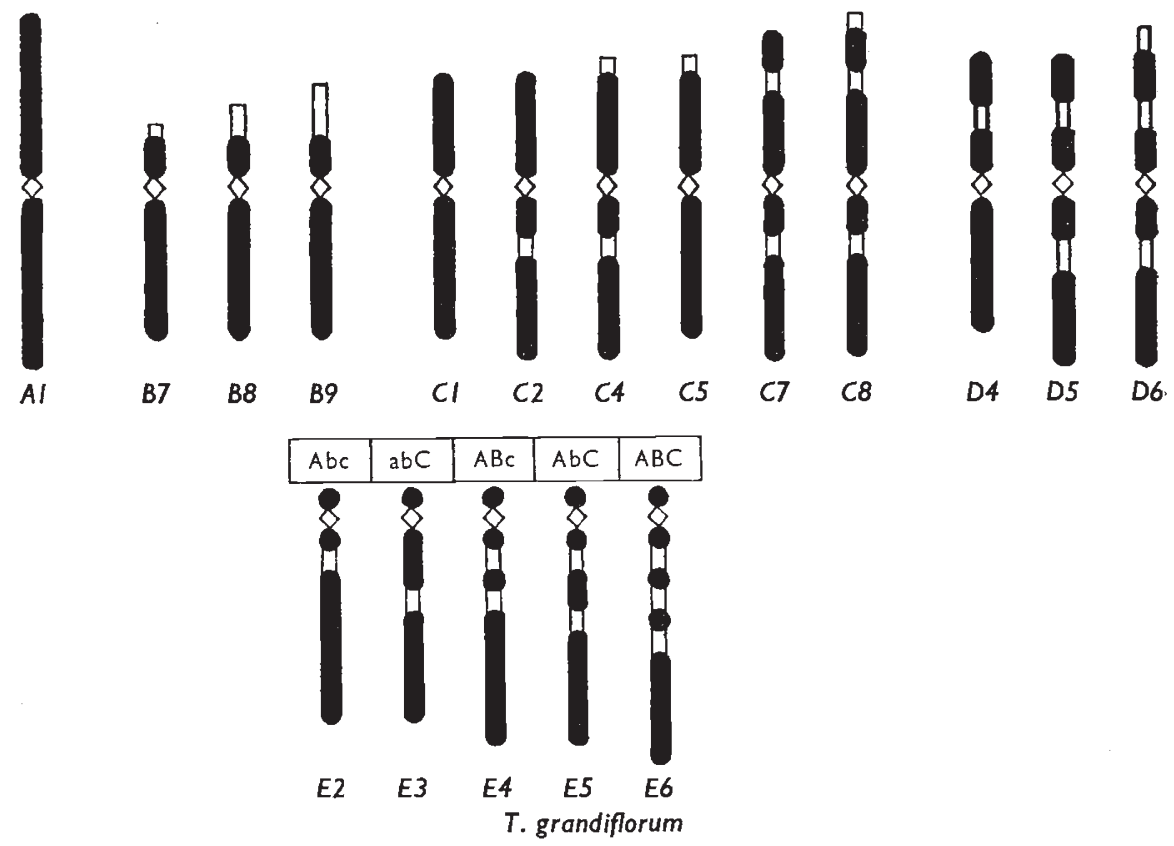

FIG. 4

5. T. grandiflorum (31 clones). Our findings agree with those of other authors.. In the root tips of our 65 plants $\mathrm{A}$ is unvarying with no $\mathrm{H}$-segments. In endosperms, 
where the coiling is not so compact as in the root, some plants have the small proximal $\mathrm{H}$-segment in the long arm of $\mathrm{A}$ also noticed by Rutishauser.

6. T. stylosum (ro clones). This species has the smallest proportion of heterochromatin (c. 2 per cent.). It is distinct from the other species only in the B and D

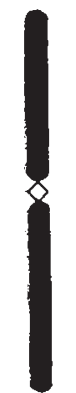

Al

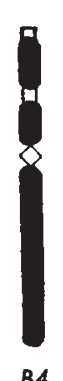

B4

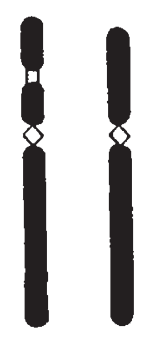

B5

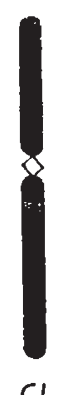

Cl

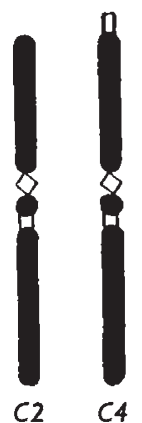

T. stylosum

Fig. 5

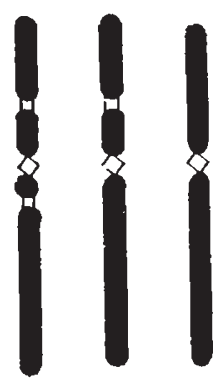

D3

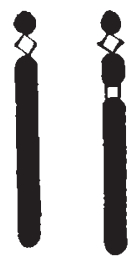

El
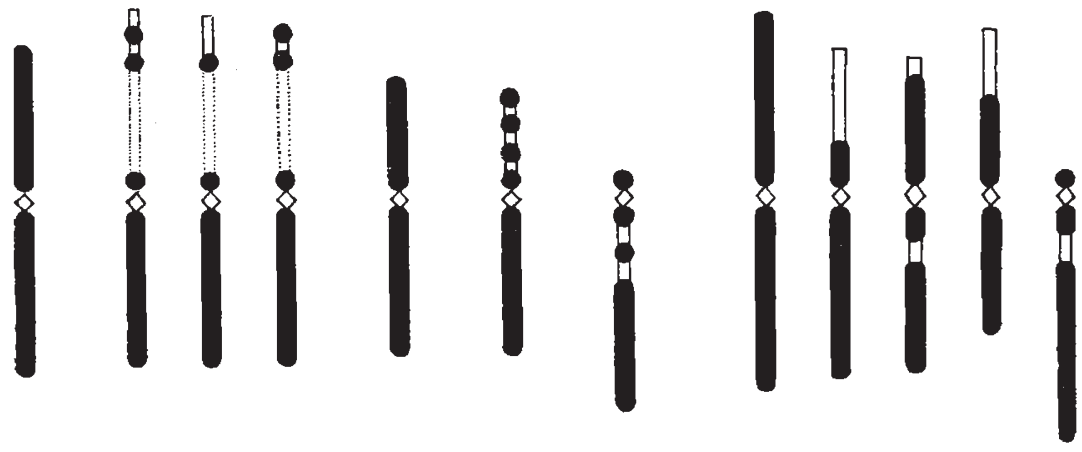

Al

BI

B2 B3 Cl

D7

E4

Al

T. recurvatum
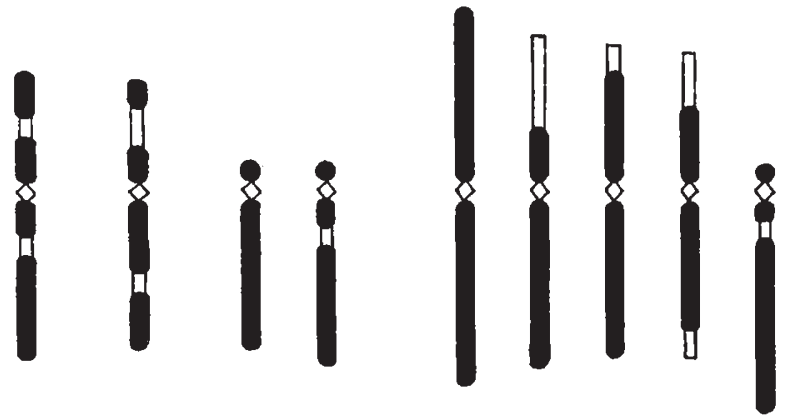

Al

$$
\begin{array}{llc}
\text { B9 } & B / 3 \quad & C 7 \\
& \text { T. ovatum }
\end{array}
$$$$
\text { D5' EI E2 }
$$$$
\text { Al }
$$

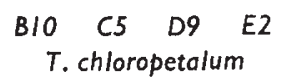

FIG. 6

chromosomes which are those developing most heterochromatin in the other species. In one plant (clone I) unique in the genus, no $\mathrm{H}$-segments could be seen after cold treatment ; two tiny chromocentres were detected in the resting nuclei.

7. T. undulatum (3 clones). In no other American species do the $\mathrm{H}$-segments reach such a high degree of differential starvation. Chromosome $B$ has a proximal 
segment in the short arm which can be seen as a starved H-segment even in unchilled metaphases. When plants are chilled, this segment fails to spiralise, and can reach up to five times its unchilled length. The length varies, depending no doubt upon how much it is stretched by movements in the cell. A non-spiralising $\mathrm{H}$-segment was first found in the $8 \mathrm{x}$ Paris japonica. It is characteristic of species like $T$. undulatum with a low H-content (Darlington and La Cour, 1940).

The distal largely euchromatic region of this arm is the sole variable in the complement of the species. It also shows the only variation that we have seen in the euchromatin of Trillium. Chromosome $\mathrm{D}_{7}$ is peculiar to this species.

8. T. ovatum. Our single clone was heterozygous in B and E. It was homozygous for $\mathrm{D}_{5}{ }^{1}$ a modification of $\mathrm{D}_{5}$ with a lengthened $\mathrm{H}$-segment.

9. T. recurvatum. Our single clone agrees with the earlier account except in being homozygous. It reaches the maximum $\mathrm{H}$-content of 25 per cent.

Io. T. chloropetalum (2 clones). The $\mathrm{H}$-pattern differs in $\mathrm{D}$ and $\mathrm{E}$ from all Trillium species and resembles $P$. polyphylla. The species is thus intermediate between the two genera. In the endosperm of a species cross a $\mathrm{D}$ chromosome revealed a small intercalary $\mathrm{H}$-segment in each arm. One of our three plants was heterozygous : E2.8.

I I. Paris polyphylla (2 clones). This species and its relatives, like Trillium kamtschaticum, have two chromosomes $\mathrm{D}$ and $\mathrm{E}$ with subterminal centromeres, and also several proximal $\mathrm{H}$-segments. These two chromosomes are also parallel in their H-pattern but whereas the $\mathrm{H}$-segments in all chromosomes are distal in Paris they are proximal in the Japanese Trillium.

Our two new clones were both different from the earlier ones although all came from Edinburgh (fig. 7).

\section{CHROMOSOME SPIRALISATION}

The chromosomes of all our species of Trillium are approximately the same size at metaphase of mitosis at normal temperatures. Those with $\mathrm{H}$-segments are of course proportionately smaller when chilled and starved. To determine the proportion of heterochromatin we need, however, to measure relative lengths which are characteristic for each clone. Now, the absolute length svary from one cell to another with the degree of spiralisation which has followed differences in the prolongation of the metaphase by colchicine. We have therefore had to calibrate our observations of different clones and species.

How was this to be done? We havefound that the lengths of euchromatin are strictly in proportion in the corresponding chromosomes of the different species. It was not difficult to find cells which closely corresponded in all species as shown in figs. $\mathrm{r}-8$. From these the composite diagram in table ro has been derived. From these also the proportions of starved heterochromatin in table $\mathrm{I}$ I have been calculated. It should be noted, however, that in the starved chromosomes the euchromatin is a little over-spiralised and the heterochromatin a little under-spiralised. As in the comparable estimate of La Cour (1951), therefore, the proportion of the unstarved length of heterochromatin would be smaller than that calculated from the starved chromosomes.

Between the American and Japanese species of Trillium a notable difference lies in the eight non-spiralising segments of $T$. kamtschaticum and its hybrids (Haga, 1956). Only one such has been found in one 
Tables 2-9.-Clonal analysis of eight species in respect of $H$-segment types ( $I-13)$ and heterozygosity of the five chromosomes $(A-E)$ and variation in supernumeraries $(f)$ of six types $\left(\mathrm{s}^{1}, \mathrm{~s}^{2}, \mathrm{t}^{0}, \mathrm{t}^{1}, \mathrm{t}^{2}, \mathrm{t}^{3}\right)$. H and $L$ mark the clones with highest and lowest $H$-segment content.

TABLE 2

T. erectum (7o Plants : 28 Clones)

\begin{tabular}{|c|c|c|c|c|c|c|c|c|}
\hline \multirow{2}{*}{$\begin{array}{c}\text { Clone } \\
\text { no. }\end{array}$} & \multicolumn{5}{|c|}{ Chromosome type and variant } & \multicolumn{2}{|c|}{ No. of plants } & \multirow{2}{*}{$\begin{array}{l}\text { No. and } \\
\text { type of } f\end{array}$} \\
\hline & A & $\mathrm{B}$ & $\mathbf{C}$ & D & $\mathrm{E}$ & No $f$ & With $f$ & \\
\hline \multicolumn{9}{|c|}{ Homozygous } \\
\hline $\begin{array}{c}1 \\
2 \\
3 \\
4 \\
5 \\
6 \\
7 \\
8 \\
9 \\
10 \\
11 \\
12 \\
13 \\
14 \\
15 \\
16 \\
17 \\
18 \\
19 \\
20 \\
21 \\
22\end{array}$ & $\begin{array}{l}2 \\
2 \\
2 \\
2 \\
2 \\
2 \\
2 \\
2 \\
2 \\
2 \\
2 \\
2 \\
2 \\
2 \\
2 \\
2 \\
3 \\
3 \\
3 \\
3 \\
3 \\
3\end{array}$ & $\begin{array}{l}\text { II } \\
\text { II } \\
\text { II } \\
\text { II } \\
\text { II } \\
\text { II } \\
\text { II } \\
\text { I2 } \\
\text { I2 } \\
12 \\
12 \\
12 \\
12 \\
12 \\
12 \\
12 \\
\text { II } \\
\text { II } \\
\text { I2 } \\
\text { I2 } \\
12 \\
\text { I2 }\end{array}$ & $\begin{array}{l}2 \\
2 \\
2 \\
6 \\
6 \\
6 \\
6 \\
2 \\
2 \\
2 \\
2 \\
4 \\
4 \\
6 \\
6 \\
6 \\
2 \\
6 \\
2 \\
4 \\
4 \\
6\end{array}$ & $\begin{array}{r}8 \\
\text { II } \\
\text { II } \\
8 \\
8 \\
\text { II } \\
\text { II } \\
8 \\
8 \\
\text { II } \\
\text { II } \\
8 \\
\text { II } \\
8 \\
\text { II } \\
\text { II } \\
8 \\
8 \\
\text { II } \\
8 \\
\text { II } \\
8\end{array}$ & $\begin{array}{l}4 \\
4 \\
6 \\
4 \\
6 \\
4 \\
6 \\
4 \\
6 \\
4 \\
6 \\
4 \\
4 \\
4 \\
4 \\
6 \\
4 \\
4 \\
6 \\
4 \\
6 \\
4\end{array}$ & $\begin{array}{l}4 \\
8 \\
I \\
5 \\
2 \\
I \\
I \\
3 \\
I \\
2 \\
4 \\
2 \\
5 \\
I \\
I \\
2 \\
3 \\
3 \\
2 \\
I \\
I \\
I\end{array}$ & $\begin{array}{c}\mathrm{I} \\
\ldots \\
\ldots \\
\ldots \\
\ldots \\
\ldots \\
\ldots \\
\ldots \\
\ldots \\
2 \\
\mathrm{I} \\
\mathrm{I} \\
\ldots \\
\mathrm{I} \\
\mathrm{I} \\
\ldots \\
\ldots \\
\ldots \\
\ldots \\
\ldots \\
\ldots \\
\ldots\end{array}$ & $\begin{array}{c}t^{1} \\
\ldots \\
\ldots \\
\ldots \\
\ldots \\
\ldots \\
\ldots \\
\ldots \\
\ldots \\
2 t^{1} \\
4 t^{0} \\
s^{1} \\
\mathrm{H} \\
6 t^{0} \\
s \\
\ldots \\
\mathrm{L} \\
\ldots \\
\ldots \\
\cdots \\
\cdots \\
\cdots \\
\cdots\end{array}$ \\
\hline \multicolumn{9}{|c|}{ Heterozygous (I pair) } \\
\hline $\begin{array}{l}23 \\
24 \\
25 \\
26 \\
27\end{array}$ & $\begin{array}{l}2 \\
2 \\
2 \\
2 \\
3\end{array}$ & $\begin{array}{l}12 \\
12 \\
12 \\
12 \\
12\end{array}$ & $\begin{array}{c}2 \cdot 4 \\
2 \cdot 4 \\
2 \cdot 6 \\
2 \\
2\end{array}$ & $\begin{array}{r}8 \\
\text { II } \\
\text { II } \\
\text { II } \\
\text { II }\end{array}$ & $\begin{array}{c}4 \\
4 \\
6 \\
4 \cdot 6 \\
4 \cdot 6\end{array}$ & $\begin{array}{l}2 \\
I \\
I \\
I \\
I\end{array}$ & $\begin{array}{c}2 \\
\ldots \\
\ldots \\
\ldots \\
\ldots\end{array}$ & $\begin{array}{l}2 t^{1} \\
\cdots \\
\cdots \\
\ldots \\
\ldots\end{array}$ \\
\hline \multicolumn{9}{|c|}{ Homozygous $\left(3^{x}\right)$} \\
\hline 28 & 2 & 12 & 2 & II & 7 & $I$ & $\cdots$ & $\ldots$ \\
\hline & & & & & Tot & $6 \mathrm{r}$ & 9 & \\
\hline
\end{tabular}


American species, T. undulatum. Small pieces of euchromatin intercalated within the non-spiralised $\mathrm{H}$-segments have enabled the Japanese workers to describe a large number of forms of each chromosome (e.g. 23 A's in T. kamtschaticum).

TABLE 3

T. cernuum (15 plants : 22 clones)

\begin{tabular}{|c|c|c|c|c|c|c|c|c|}
\hline \multirow{2}{*}{$\begin{array}{l}\text { Clone } \\
\text { no. }\end{array}$} & \multicolumn{5}{|c|}{ Chromosome type and variant } & \multicolumn{2}{|c|}{ No. of plants } & \multirow{2}{*}{$\begin{array}{l}\text { No. and } \\
\text { type of } f\end{array}$} \\
\hline & A & B & C & D & $\mathrm{E}$ & No $f$ & With $f$ & \\
\hline$I$ & 2 & I I & 4 & II & 4 & 3 & $\ldots$ & \\
\hline 2 & 2 & II & $\begin{array}{l}\mathrm{T} \\
4\end{array}$ & II & 6 & $I$ & $\ldots$ & $\dddot{H}$ \\
\hline 3 & 2 & I I & 5 & II & 4 & $I$ & $\ldots$ & $\ldots$ \\
\hline 4 & 2 & 13 & 4 & I I & 4 & $I$ & $\ldots$ & $\ldots$ \\
\hline 5 & 2 & I3 & 5 & I I & 4 & $I$ & $\cdots$ & $\cdots$ \\
\hline 6 & 3 & II & 4 & II & 4 & 6 & $\ldots$ & $\ldots$ \\
\hline 7 & 3 & II & 4 & II & 6 & 2 & $\ldots$ & $\cdots$ \\
\hline 8 & 3 & I I & 5 & I I & 4 & 5 & $\ldots$ & $\ldots$ \\
\hline 9 & 3 & I 3 & 2 & II & 4 & $I$ & $\ldots$ & L \\
\hline IO & 3 & I3 & 4 & II & 4 & 5 & $\ldots$ & $\ldots$ \\
\hline$I I$ & 3 & 13 & 4 & II & 6 & $I$ & $\cdots$ & $\cdots$ \\
\hline 12 & 3 & 13 & 5 & Io & 4 & $I$ & $\ldots$ & $\ldots$ \\
\hline 13 & 3 & I3 & 5 & II & 4 & 6 & $\cdots$ & $\cdots$ \\
\hline \multicolumn{9}{|c|}{ Heterozygous (I pair) } \\
\hline$I_{4}$ & 3 & & 5 & & & $I$ & $\ldots$ & $\ldots$ \\
\hline 15 & 3 & 13 & $2 \cdot 4$ & I I & 4 & $\ldots$ & 2 & $2 t$ \\
\hline 16 & 3 & II & 4 & $10 \cdot 11$ & 6 & $I$ & $\ldots$ & $\ldots$ \\
\hline 17 & 2 & I I & 5 & Io & $4 \cdot 6$ & $I$ & $\ldots$ & $\ldots$ \\
\hline 18 & 3 & II & 2 & I I & $4 \cdot 6$ & $I$ & $\ldots$ & $\ldots$ \\
\hline 19 & 3 & I I & 4 & I I & $4 \cdot 6$ & $I$ & $\cdots$ & $\ldots$ \\
\hline 20 & 3 & I I & 4 & I I & $4 \cdot 6$ & 2 & $\ldots$ & $\ldots$ \\
\hline $2 I$ & 3 & 13 & 2 & II & $4 \cdot 6$ & $I$ & $\ldots$ & $\ldots$ \\
\hline 22 & 3 & I3 & 5 & II & $4 \cdot 6$ & $I$ & $\cdots$ & $\cdots$ \\
\hline & & & & & Total & 43 & 2 & \\
\hline
\end{tabular}

\section{VARIATION IN H-SEGMENTS}

\section{(i) Species}

The five basic chromosomes of Trillium are fairly distinct throughout the American species in their form combined with their pattern of $\mathrm{H}$-segments (figs. I-8). This pattern varies between species. But, in all our samples that include more than three plants, it also varies within species. This variation appears almost entirely as variation in the number, position and size of the H-segments.

In our whole collection the proportion of heterochromatin varies from 0.5 to 25 per cent. of the chromosome length. Within each species the range is smaller (table II). Euchromatin may also vary as we see in the $B_{1}, B_{2}, B_{3}$ series where the small blocks expose the 
TABLE 4

T. luteum (3o plants : 28 clones)

\begin{tabular}{|c|c|c|c|c|c|c|c|c|}
\hline \multirow{2}{*}{$\begin{array}{c}\text { Clone } \\
\text { No. }\end{array}$} & \multicolumn{5}{|c|}{ Chromosome type and variant } & \multicolumn{2}{|c|}{ No. of plants } & \multirow{2}{*}{$\begin{array}{l}\text { No. and } \\
\text { type of } f\end{array}$} \\
\hline & $\mathrm{A}$ & $\mathrm{B}$ & C & $\mathrm{D}$ & $\mathbf{E}$ & No $f$ & With $f$ & \\
\hline \multicolumn{9}{|c|}{ Homozygous } \\
\hline$I$ & I & 8 & 2 & 4 & I & $\cdots$ & 3 & $t$ \\
\hline 2 & I & 8 & 2 & 5 & 2 & ... & I & $t$ \\
\hline 3 & I & 9 & 2 & 5 & 4 & $\ldots$ & I & $s^{1}$ \\
\hline 4 & I & IO & 2 & 5 & I & $I$ & $\cdots$ & $\cdots$ \\
\hline \multicolumn{9}{|c|}{ Heterozygous for I pair } \\
\hline 5 & I & $6 \cdot 8$ & 2 & 4 & I & $I$ & $\cdots$ & $\ldots \mathrm{L}$ \\
\hline 6 & I & $6 \cdot 9$ & 2 & 5 & I & $I$ & $\cdots$ & $\cdots$ \\
\hline 7 & I & $6 \cdot 10$ & 3 & 5 & I & $I$ & $\cdots$ & $\cdots$ \\
\hline 8 & I & $7 \cdot 8$ & 2 & 4 & 3 & $I$ & $\cdots$ & $\cdots$ \\
\hline 9 & I & $7 \cdot 9$ & 2 & 5 & I & $I$ & $\cdots$ & $\cdots$ \\
\hline $1 O$ & I & $8 \cdot 9$ & 2 & 5 & I & $\cdots$ & I & $s$ \\
\hline$I I$ & I & $8 \cdot 9$ & 3 & 5 & 2 & $\cdots$ & I & $s$ \\
\hline 12 & I & $8 \cdot 10$ & 2 & 4 & 2 & $\cdots$ & I & $2 t$ \\
\hline 13 & I & $8 \cdot 10$ & 2 & 5 & 2 & $\cdots$ & I & ${ }^{t} \mathrm{H}$ \\
\hline 14 & I & $9 \cdot 10$ & 4 & 5 & 3 & $I$ & ${ }_{I}$ & ${ }_{2 s} \mathrm{H}$ \\
\hline 15 & I & $\begin{array}{l}8 \\
8\end{array}$ & 2.4 & 5 & I & $\begin{array}{l}\ldots \\
\ldots\end{array}$ & $\begin{array}{l}1 \\
\text { I }\end{array}$ & $t$ \\
\hline 16 & $\begin{array}{l}\text { I } \\
\text { I }\end{array}$ & $\begin{array}{l}8 \\
9\end{array}$ & $\begin{array}{l}2.4 \\
2.2\end{array}$ & $\begin{array}{l}5 \\
4\end{array}$ & $\begin{array}{l}\text { I } \\
\text { I }\end{array}$ & $\cdots$ & I & $t$ \\
\hline $\begin{array}{l}17 \\
18\end{array}$ & & $\begin{array}{r}9 \\
10\end{array}$ & $\begin{array}{l}2 \cdot 3 \\
2 \cdot 4\end{array}$ & $\begin{array}{l}4 \\
5\end{array}$ & 2 & $I$ & $\ldots$ & $\ldots$ \\
\hline 19 & I & 8 & 2 & $2 \cdot 4$ & 3 & $\ldots$ & I & $s$ \\
\hline 20 & I & 7 & 2 & 4 & $\mathrm{I} \cdot 3$ & $\ldots$ & I & $s$ \\
\hline $2 I$ & I & 8 & 3 & 5 & $2 \cdot 3$ & $I$ & $\ldots$ & $\cdots$ \\
\hline 22 & I & 10 & 2 & 5 & $I \cdot 2$ & $I$ & $\cdots$ & $\cdots$ \\
\hline \multicolumn{9}{|c|}{ Heterozygous for 2 pairs } \\
\hline 23 & I & $7 \cdot 8$ & $2 \cdot 4$ & 4 & 2 & $\cdots$ & I & $t$ \\
\hline 24 & I & $7 \cdot 9$ & $2 \cdot 4$ & 5 & 3 & $I$ & $\cdots$ & $\ldots$ \\
\hline 25 & I & $8 \cdot 9$ & 2 & 5 & $2 \cdot 3$ & $\cdots$ & I & $s$ \\
\hline 26 & I & 8 & 3 & $4 \cdot 5$ & I. 4 & $I$ & $\cdots$ & $\cdots$ \\
\hline \multicolumn{9}{|c|}{ Heterozygous for 3 pairs } \\
\hline 27 & I & $6 \cdot 8$ & $2 \cdot 4$ & 4 & I. 3 & $I$ & $\ldots$ & $\ldots$ \\
\hline 28 & $I$ & $8 \cdot 9$ & $2 \cdot 3$ & 4 & $2 \cdot 3$ & $I$ & $\cdots$ & $\cdots$ \\
\hline \multicolumn{9}{|c|}{ Total } \\
\hline
\end{tabular}


variation to our view. But it is obviously less variable in quantity than the heterochromatin.

Now the variation in the $\mathrm{H}$-segments follows a similar pattern in different species : the polymorphism is parallel not only in each

TABLE 5

T. sessile (20 plants : 13 clones)

\begin{tabular}{|c|c|c|c|c|c|c|c|c|}
\hline \multirow{2}{*}{$\begin{array}{c}\text { Clone } \\
\text { no. }\end{array}$} & \multicolumn{5}{|c|}{ Chromosome type and variant } & \multicolumn{2}{|c|}{ No. of plants } & \multirow{2}{*}{$\begin{array}{l}\text { No. and } \\
\text { type of } f\end{array}$} \\
\hline & A & B & C & $\mathrm{D}$ & $\mathbf{E}$ & No $f$ & With $f$ & \\
\hline \multicolumn{9}{|c|}{ Homozygous } \\
\hline$I$ & I & 9 & I & 4 & I & $I$ & $\ldots$ & $\ldots$ \\
\hline 2 & I & 9 & 2 & 4 & I & $I$ & 4 & $s, 3^{s}$ \\
\hline 3 & I & 10 & 2 & 4 & I & $\ldots$ & 1 & $s, \mathrm{H}$ \\
\hline \multicolumn{9}{|c|}{ Heterozygous for I pair } \\
\hline 4 & I & $6 \cdot 9$ & 2 & 4 & I & $\ldots$ & I & $s \mathrm{~L}$ \\
\hline $\begin{array}{l}7 \\
5\end{array}$ & I & 7.9 & I & 4 & I & $I$ & I & $s$ \\
\hline 6 & I & $7 \cdot 9$ & 2 & 4 & I & $\ldots$ & I & $s$ \\
\hline 7 & I & $7 \cdot 10$ & 2 & 4 & I & $\ldots$ & I & $s$ \\
\hline 8 & I & $9 \cdot 10$ & I & 4 & I & $I$ & $\ldots$ & $\ldots$ \\
\hline 9 & $I$ & $9 \cdot 10$ & 2 & 4 & I & $I$ & 2 & $s$ \\
\hline IO & I & 9 & I & $4 \cdot 5$ & I & $I$ & $\ldots$ & $\ldots$ \\
\hline$I I$ & I & 9 & 2 & 4.5 & I & $\ldots$ & I & $2 s$ \\
\hline \multicolumn{9}{|c|}{ Heterozygous for 2 pairs } \\
\hline 12 & I & 7.9 & 2 & $4 \cdot 5$ & I & $\ldots$ & I & $t$ \\
\hline 12 & I & $7 \cdot 9$ & 2 & 4 & $1 \cdot 2$ & $\cdots$ & I & $2 s$ \\
\hline \multicolumn{9}{|c|}{ Total } \\
\hline
\end{tabular}

chromosome but in each arm of a chromosome. For example, the two arms of A are always similar, those of B always widely different. Each chromosome has its characteristic range and also its occasional unique variant. This parallelism would, no doubt, be more striking if more plants and more populations were studied.

How does this parallelism come about? It could have arisen in three ways :

First, free recombination might be occurring between species whose supposed differences were no barrier to crossing. This, however, seems to be excluded in Trillium by clear separation of many of our species. The results of species crossing are known only in polyploids found by Haga (1956) in Japan, but more hybridisation should be attempted. 
TABLE 6

T. grandiflorum (65 plants: $3^{I}$ clones)

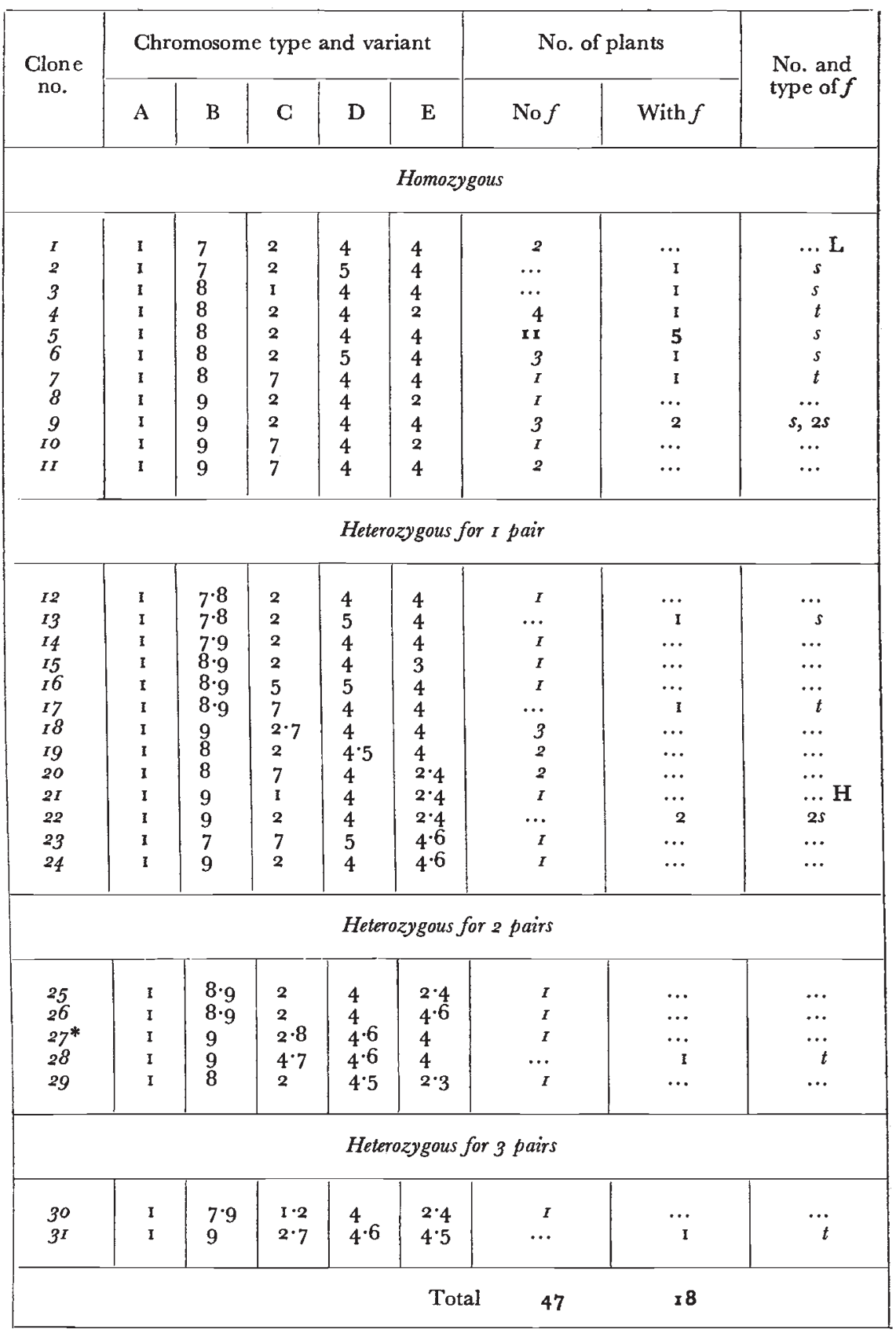

* Plant T.g. 27 presents us with a problem in the distinction of C and D. It is here assumed to be heterozygous for the type C8. This plant is, however, also heterozygous for D6. We are now inclined to suppose that both these chromosomes are of the unique type $\mathrm{C} 8$ and that the $\mathrm{C}_{2}$ is a new type of $\mathrm{D}$ which we might number Di2. The formula of the plant would then be C8.8, $\mathrm{D}_{4} .12$. 
POLYMORPHISM OF HETEROGHROMATIN IN TRILLIUM IOI TABLE 7

T. stylosum (20 plants : Io clones)

\begin{tabular}{|c|c|c|c|c|c|c|c|c|}
\hline \multirow{2}{*}{$\begin{array}{c}\text { Clone } \\
\text { no. }\end{array}$} & \multicolumn{5}{|c|}{ Chromosome type and variant } & \multicolumn{2}{|c|}{ No. of plants } & \multirow{2}{*}{$\begin{array}{l}\text { No. and } \\
\text { type of } f\end{array}$} \\
\hline & A & B & C & $\mathrm{D}$ & $\mathbf{E}$ & Nof & With $f$ & \\
\hline \multicolumn{9}{|c|}{ Homozygous } \\
\hline$I$ & I & 4 & 2 & 2 & I & $I$ & $\cdots$ & $\cdots$ \\
\hline 2 & I & 5 & I & 3 & 2 & $I$ & ... & ... \\
\hline 3 & I & 5 & I & 2 & I & 2 & ... & $\cdots$ \\
\hline 4 & I & 5 & $\mathbf{I}$ & 2 & 2 & 2 & $\cdots$ & $\ldots$ \\
\hline 5 & I & 5 & 2 & 2 & 2 & 5 & ... & $\cdots$ \\
\hline 6 & I & 5 & 2 & I & 2 & $I$ & $\cdots$ & $\cdots$ \\
\hline 7 & I & 5 & 2 & 2 & I & $I$ & $\cdots$ & $\cdots$ \\
\hline 8 & I & 5 & 2 & 3 & 2 & 5 & $\cdots$ & $\cdots$ \\
\hline 9 & I & 6 & I & 3 & I & $I$ & $\cdots$ & $\cdots$ \\
\hline \multicolumn{9}{|c|}{ Heterozygous for I pair } \\
\hline IO & I & 5 & $2 \cdot 4$ & 3 & 2 & $I$ & $\cdots$ & $\mathrm{H}$ \\
\hline \multicolumn{9}{|c|}{ Total } \\
\hline
\end{tabular}

TABLE 8

T. undulatum (20 plants : 3 clones)

\begin{tabular}{|c|c|c|c|c|c|c|c|c|}
\hline \multicolumn{9}{|c|}{ Homozygous } \\
\hline $\begin{array}{l}I \\
2 \\
3\end{array}$ & $\begin{array}{l}\text { I } \\
\text { I } \\
\text { I }\end{array}$ & $\begin{array}{l}\text { I } \\
2 \\
3\end{array}$ & $\begin{array}{l}\text { I } \\
\text { I } \\
\text { I }\end{array}$ & $\begin{array}{l}7 \\
7 \\
7\end{array}$ & $\begin{array}{l}4 \\
4 \\
4\end{array}$ & $\begin{array}{l}3 \\
4 \\
3\end{array}$ & $\begin{array}{c}\ldots \\
\ldots \\
\ldots\end{array}$ & $\begin{array}{c}\dddot{H} \\
\mathrm{~L}\end{array}$ \\
\hline \multicolumn{9}{|c|}{ Total } \\
\hline
\end{tabular}

TABLE 9

(P. polyphylla (9 plants : 3 clones)

\begin{tabular}{|l|l|l|l|l|l|l|l|l|l|}
\hline \multicolumn{10}{|c|}{ Heterozygous } \\
\hline$I^{*}$ & $\mathrm{I}$ & 2 & $\mathrm{I} \cdot 2$ & $\mathrm{I}$ & $\mathrm{I}$ & $\ldots$ & $\mathrm{I}$ & $s$ \\
2 & $\mathrm{I}$ & $\mathrm{I} \cdot 2$ & 2 & 2 & 2 & 4 & $\mathrm{I}$ & 5 \\
3 & $\mathrm{I}$ & $\mathrm{I} \cdot 3$ & 2 & 3 & 3 & $\ldots$ & 3 & 5 \\
\hline
\end{tabular}

G 2

* Plant described by Darlington and La Cour (1938). 
Secondly, pre-determining conditions might have led to parallel development of $\mathrm{H}$-segments after the separation of the species. There

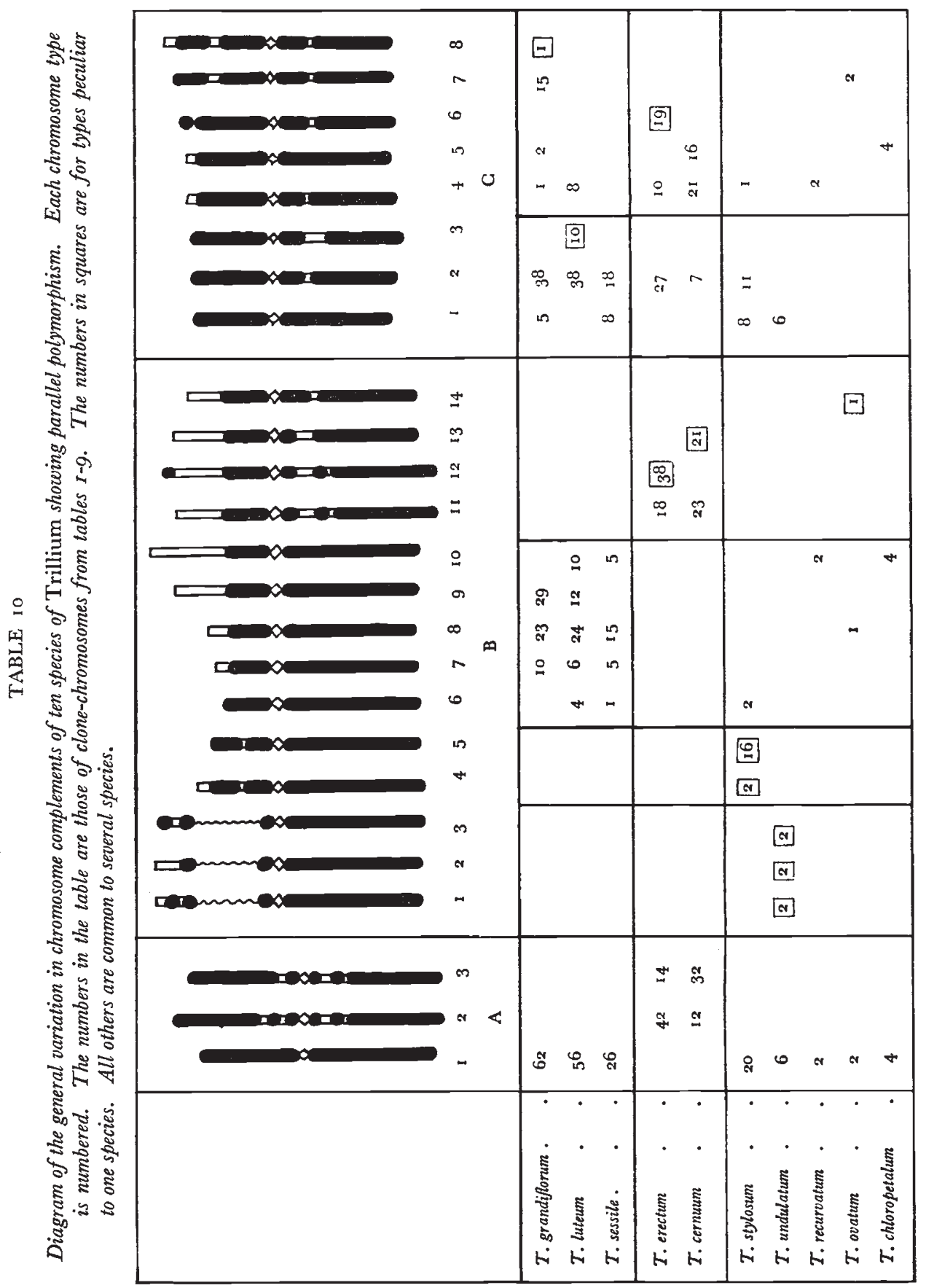

are, of course, conditions limiting the development of $\mathrm{H}$-segments. For example, although of the eighty-eight ends of the forty-four 
POLYMORPHISM OF HETEROGHROMATIN IN TRILLIUM 103

chromosome variants twenty were heterochromatic only one variant had $\mathrm{H}$-segments at both ends. There are, therefore, principles limiting

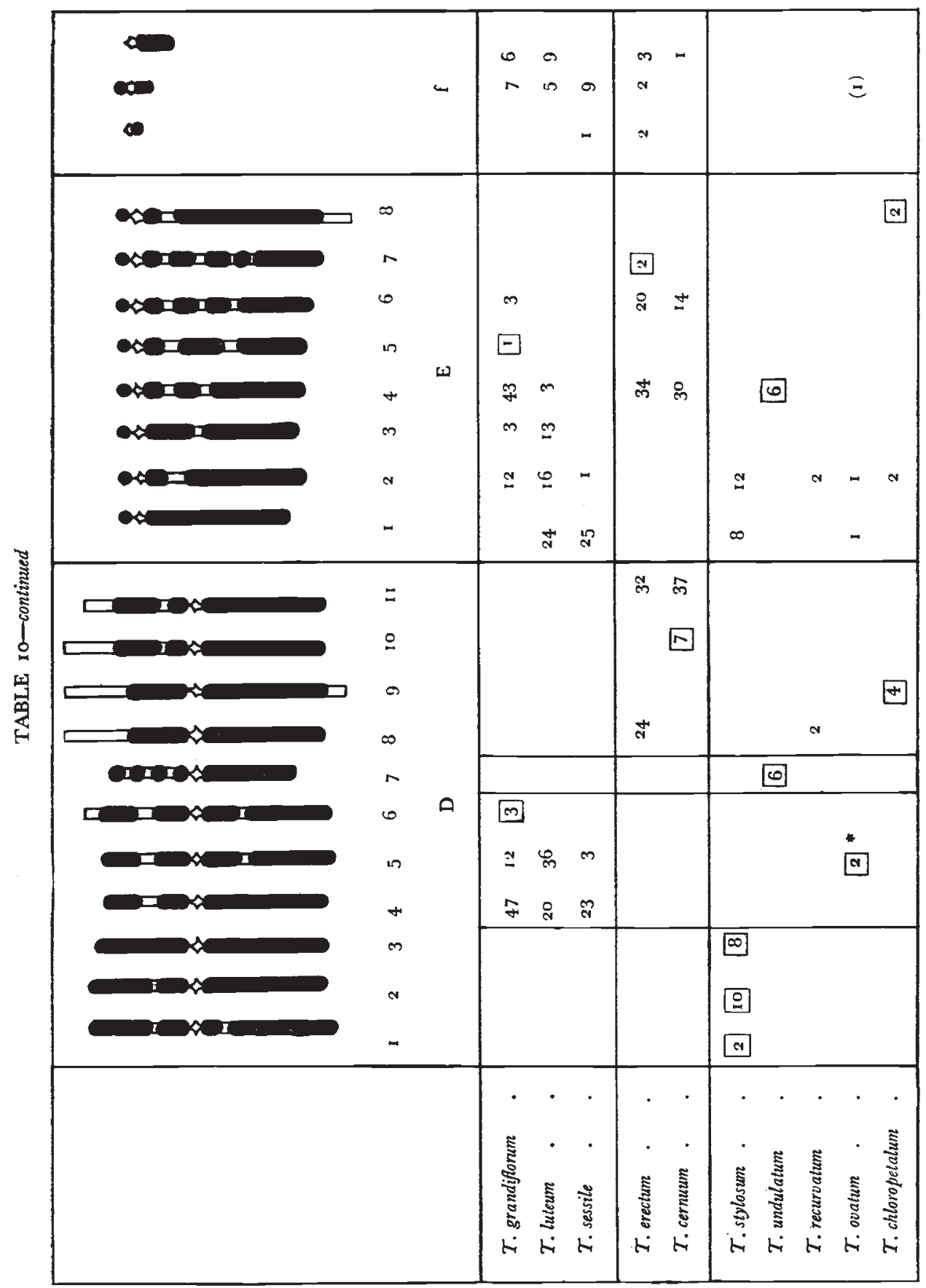

the distribution of heterochromatin and probably equalising its distribution within and between the chromosomes perhaps to meet 
the mechanical needs of mitosis or the physiological needs of the resting nucleus.

\section{TABLE II}

Variation and heterozygosity among different chromosomes in species with the larger samples, indicating the range of heterochromatin content in the chilled chromosomes ( $L$ to $H$ )

\begin{tabular}{|c|c|c|c|c|c|c|c|}
\hline \multirow{2}{*}{\multicolumn{2}{|c|}{ Species }} & & \multirow{2}{*}{$\begin{array}{c}\text { No } \\
\text { variants }\end{array}$} & \multicolumn{2}{|c|}{ With variants } & \multirow{2}{*}{\multicolumn{2}{|c|}{$\begin{array}{l}\text { Proportion of } \\
\text { heterochromatin } \\
\text { (per cent.) } \\
\text { L } \quad \text { H }\end{array}$}} \\
\hline & & & & No heterozygotes & Heterozygotes & & \\
\hline $\int \mathcal{T}$ erectum & . & - & $\ldots$ & $\begin{array}{lllll}A & B & \ldots & D & \ldots\end{array}$ & $\begin{array}{lllll}\ldots & \ldots & \mathrm{G} & \ldots & \mathrm{E}\end{array}$ & 16 & 19 \\
\hline$\{T$. cernuum & . & - & $\cdots$ & A $\ldots \ldots \ldots c$ & $\ldots B \quad C \quad D \quad E$ & 12 & 15 \\
\hline T. grandiflor & & . & A & 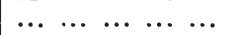 & $\ldots B \quad C \quad D \quad E$ & 6 & 10 \\
\hline T. luteum & . & . & A & $\begin{array}{lllllllll} & \ldots & \ldots & \ldots & \ldots & \ldots\end{array}$ & $\ldots$ B $\quad$ C $\quad D \quad E$ & 3 & 9 \\
\hline T. sessile & . & . & A & 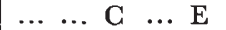 & $\begin{array}{lllll}\ldots & \text { B } & \ldots & \text { D } & \ldots\end{array}$ & 5 & 8 \\
\hline T. stylosum & . & . & A & $\begin{array}{llll}\ldots & B & C & D\end{array}$ & $\begin{array}{llllll}\ldots & \ldots & \ldots & \ldots & \ldots\end{array}$ & 0.5 & 2 \\
\hline
\end{tabular}

These general conditions, however, can hardly have decided the exact repetitions of pattern in different species. We prefer, therefore, the third possibility that the $\mathrm{H}$-segments vary in parallel on account

TABLE 12

Frequency of structurally heterozygous pairs in relation to the number of variants of the five chromosome types in each species of Trillium and Paris

\begin{tabular}{|c|c|c|c|c|c|c|c|c|}
\hline Species & & $\begin{array}{l}\text { No. of } \\
\text { clones }\end{array}$ & A B & $\mathrm{C}$ & D E & Total & $\begin{array}{c}\text { Heterozygous } \\
\text { pairs } \\
\text { (per cent.) }\end{array}$ & $\begin{array}{c}\text { No. of } \\
\text { variants* }\end{array}$ \\
\hline $\begin{array}{l}\text { T. grandiflorum } \\
\text { T. luteum . } \\
\text { T. sessile } \\
\text { T. cernuum } \\
\text { T. erectum. } \\
\text { T. stylosum } \\
\text { T. ovatum } \\
P . \text { polyphylla }{ }^{\dagger}\end{array}$ & $: \quad:$ & $\begin{array}{r}31 \\
28 \\
13 \\
22 \\
28 \\
10 \\
1 \\
4\end{array}$ & $\begin{array}{lr}\ldots & 9 \\
\ldots & 15 \\
\ldots & 8 \\
\ldots & 1 \\
\ldots & \ldots \\
\ldots & \ldots \\
\ldots & 1 \\
\ldots & \ldots\end{array}$ & $\begin{array}{r}5 \\
8 \\
\cdots \\
1 \\
3 \\
1 \\
\cdots \\
3\end{array}$ & $\begin{array}{rr}5 & 10 \\
2 & 7 \\
3 & 1 \\
1 & 6 \\
\ldots & 2 \\
\ldots & \ldots \\
\ldots & 1 \\
\ldots & \ldots\end{array}$ & $\begin{array}{r}29 \\
32 \\
12 \\
9 \\
5 \\
1 \\
2 \\
3\end{array}$ & $\begin{array}{l}18 \cdot 7 \\
22 \cdot 8 \\
17 \\
8 \cdot 2 \\
3 \cdot 6 \\
2 \\
\ldots \\
15\end{array}$ & $\begin{array}{r}13 \\
10 \\
6 \\
6 \\
7 \\
7 \\
2 \\
4\end{array}$ \\
\hline Total & . & $13^{8}$ & $\cdots 34$ & 21 & $\begin{array}{ll}11 & 27\end{array}$ & 93 & $\ldots$ & $\ldots$ \\
\hline
\end{tabular}

* Variants are the numbers of types in excess of one for each of the five chromosomes.

$\dagger$ Includes one clone previously described.

of common descent from a similarly polymorphic ancestor whosevariation has been preserved in the ten species. Thus we infer a very ancient and stable polymorphism depending on a conservation of the linear structure of the chromosome in respect of heterochromatin, or at least of intercalary heterochromatin.

This conclusion is in keeping with the general conservatism seen. in the basic set of five chromosomes of Trillium. 
(ii) Populations

The populations being variable we naturally wish to find out the frequency of heterozygotes for the variant chromosome types. The species-samples (or populations) with the most variants are of course,

TABLE I3

Frequencies of different types of particular chromosomes in combinations of pairs of homologues*

(i) Trillium erectum and $T$. cernuum

Difference in frequency of heterozygotes between $\mathrm{A}$ and $\mathrm{E}$ chromosomes.

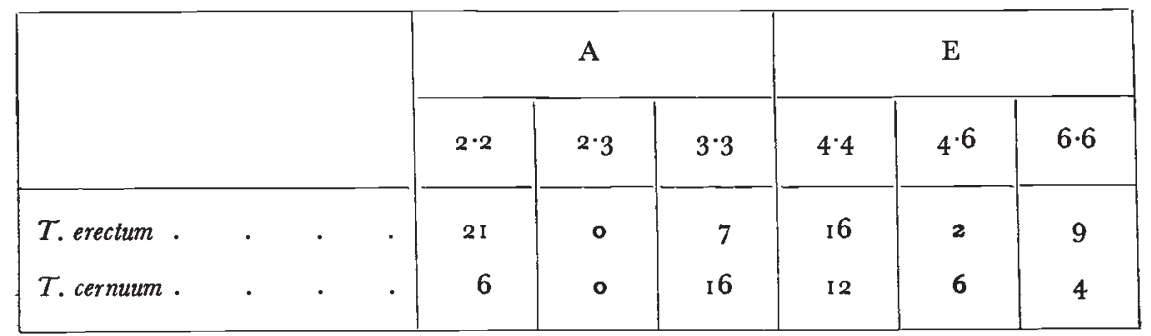

(ii) Trillium luteum : $\mathrm{B}, 5$ types

( 56 chromosomes of 28 clones : cf. table 4 )

\begin{tabular}{|c|c|c|c|c|c|}
\hline B & 6 & 7 & 8 & 9 & IO \\
\hline 6 & $\begin{array}{c}\ldots \\
(0 \cdot 15)\end{array}$ & & 2 & I & I \\
\hline 7 & & $\begin{array}{c}\mathrm{I} \\
(0 \cdot 32)\end{array}$ & 2 & 2 & $\cdots$ \\
\hline 8 & & & $\begin{array}{c}7 \\
(5 \cdot I)\end{array}$ & 4 & 2 \\
\hline 9 & & & & $\begin{array}{c}2 \\
(I \cdot 25)\end{array}$ & I \\
\hline IO & & & & & $(o \cdot \stackrel{3}{90})$ \\
\hline Total & 4 & 6 & 24 & 12 & 10 \\
\hline
\end{tabular}

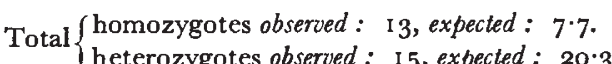

* In table 13 the chromosome types are put in ascending order of H-content, i.e. the highest values are reached in $\mathrm{Br}, \mathrm{C} 8$ and $\mathrm{E}_{5}$. The modal frequency is always at an intermediate content.

in general, those with the largest proportion of heterozygotes (table 12). Heterogeneity is a condition of heterozygosity - a principle Haga and Kurabayashi (1954) seem to have overlooked.

This frequency, it seems, is rarely equal to, and usually lower than the expectation with random recombination. But the deficiency of heterozygotes seems to differ for different chromosomes in the same plants from one population (table i3 (i)).

Taking the populations with the highest frequency of heterozygotes we have compared our observations with expectation on an assumption of random recombination and unselected survival (table 
I3 (ii)-(iv)). The results show that all possible combinations between and within chromosomes may well occur, as they should do in a freely

TABLE I 3-continued

(iii) Trillium grandiflorum: C, 6 types

(62 chromosomes of $3^{1}$ clones : $c f$. table 6$)$

\begin{tabular}{|c|c|c|c|c|c|c|}
\hline C & $I$ & 5 & 2 & 4 & 7 & 8 \\
\hline & $\begin{array}{c}2 \\
(0 \cdot 2)\end{array}$ & & I & & & \\
\hline 5 & & $\left(\begin{array}{c}1 \\
\left(0.03^{2}\right)\end{array}\right.$ & $\cdots$ & & $\cdots$ & $\cdots$ \\
\hline 2 & & & $\begin{array}{c}17 \\
(I I \cdot 7)\end{array}$ & $\ldots$ & 2 & I \\
\hline & & & & $(0.008)$ & I & $\cdots$ \\
\hline 7 & & & & & $\begin{array}{c}6 \\
(r \cdot 8)\end{array}$ & $\cdots$ \\
\hline 8 & & & & & & $\left(\begin{array}{c}\cdots \\
(0.008)\end{array}\right.$ \\
\hline Total & 5 & 2 & $3^{8}$ & I & I5 & I \\
\hline
\end{tabular}

Total $\left\{\begin{array}{l}\text { homozygotes observed: } 26 \text {, expected : } 13.7 . \\ \text { heterozygotes observed : } 36 \text {, expected : } 48 \cdot 3 \text {. }\end{array}\right.$

Note.- $\mathrm{C}_{2}$ is the commonest chromosome type as measured by our clones. Since it is two of our homozygous C2.2 clones that are represented by the largest numbers of plants ( 16 and 5 ) the clones may in fact be composed of identical seedlings.

(iv) Trillium grandiflorum: E, 5 types (62 chromosomes of 31 clones)

\begin{tabular}{|c|c|c|c|c|c|}
\hline $\mathrm{E}$ & $I$ & 3 & 2 & 4 & 5 \\
\hline$I$ & $\begin{array}{c}3 \\
(r \cdot 2)\end{array}$ & & & & $\cdots$ \\
\hline 3 & & $\begin{array}{c}17 \\
(15 \cdot 0)\end{array}$ & & & 3 \\
\hline 2 & & & $\left(\begin{array}{c}I \\
(0.07)\end{array}\right.$ & $\cdots$ & $\cdots$ \\
\hline 4 & & & & $(0.008)$ & $\cdots$ \\
\hline 5 & & & & & $(0.07)$ \\
\hline Total & 12 & 43 & 3 & $I$ & 3 \\
\hline
\end{tabular}

Total $\left\{\begin{array}{l}\text { homozygotes observed : } 2 \mathrm{I} \text {, expected : } 16 \cdot 4 . \\ \text { heterozygotes observed : } 4 \mathrm{I} \text {, expected }: 45.6\end{array}\right.$

interbreeding population. But within chromosomes, homozygotes are again too numerous : in one sample only ( $\mathrm{E}$ of $\mathcal{T}$. grandiflorum) is there no serious deficiency of heterozygotes. Even here there would be serious deficiency if we admitted, what is most likely, that our multiple clone with sixteen plants (No. 5) was, in fact, sixteen seedlings. 
The lack of apparent advantage of heterozygotes implies that even the heterozygosity of euchromatin adjoining the H-segments is not

TABLE 14

Combinations of chromosomes which are assumed to recombine two differences by crossing over in populations with different frequencies of heterozygotes in respect of the relevant chromosomes, compared with random expectation.

(i) T. cernuum: 22 clones, table 3

\begin{tabular}{|c|c|c|c|c|c|}
\hline $\mathrm{C}$ & $I$ & 4 & 2 & 5 & $\mathrm{~T}$ \\
\hline $\mathrm{ab} \quad I$ & $\ldots$ & $\ldots$ & $\ldots$ & $\cdots$ & \\
\hline $\mathrm{AB} \quad 4$ & & 10 & $I$ & $\ldots$ & \\
\hline $\mathrm{aB} \quad 2$ & & & 3 & $\ldots$ & \\
\hline $\mathrm{Ab} \quad 5$ & & & & 8 & \\
\hline Total & $\ldots$ & $2 I$ & 7 & 16 & 44 \\
\hline Expected & $2 \cdot 5$ & $23 \cdot 5$ & $4 \cdot 5$ & 13.5 & \\
\hline
\end{tabular}

(ii) T. grandiforum: $3^{1}$ clones, tables 6,13 (iii)

\begin{tabular}{|c|c|c|c|c|c|}
\hline C & $I$ & $4+8$ & $2+7$ & 5 & $\mathrm{~T}$ \\
\hline$a b$ & 2 & $\ldots$ & 1 & $\ldots$ & \\
\hline $\mathrm{AB} \quad 4+8$ & & $\cdots$ & 2 & $\ldots$ & \\
\hline $\mathrm{aB} \quad 2+7$ & & & 25 & $\ldots$ & \\
\hline $\mathrm{Ab} \quad 5$ & & & & 1 & \\
\hline Total & 5 & 2 & 53 & 2 & 62 \\
\hline Expected & $6 \cdot 55$ & $3 \cdot 55$ & $5^{I} \cdot 45$ & 0.45 & \\
\hline
\end{tabular}

being favoured by selection. This situation is not unknown but it is in contrast to a common experience of inversion, interchange and fragmentation heterozygotes where euchromatin alone is in question.

The tables of Haga for his Sizunai population of $T$. kamtschaticum have been treated in the same way by our colleague, Mr A. F. Dyer (unpublished). He finds that homozygotes are in excess where larger numbers of chromosome types are present (in A, C and D). But in $\mathrm{B}$ and $\mathrm{E}$, where there are few chromosome types and one or two are preponderant, the random heterozygote frequency is reached.

There is, however, a second kind of test that we can apply to these populations. 
Intercalary $\mathrm{H}$-segments can be recombined by crossing over as Rutishauser (1956b) has shown from the endosperm of Trillium grandiflorum. In three species we find pairs of chromosomes which differ in respect of the presence and absence of pairs of $\mathrm{H}$-segments, giving four types of chromosome (table r 4 ).

TABLE 14-continued

(iii) T. luteum : 28 clones, table 4

\begin{tabular}{|c|c|c|c|c|c|}
\hline $\mathrm{E}$ & $I$ & 4 & 2 & 3 & $\mathrm{~T}$ \\
\hline$a b$ & 10 & I & 1 & 2 & \\
\hline $\mathrm{AB}$ & & I & $\ldots$ & $\ldots$ & \\
\hline $\mathrm{Ab}$ & & & 6 & 3 & \\
\hline $\mathrm{aB}$ & & & & 4 & \\
\hline Total & 24 & 3 & I6 & I3 & $5^{6}$ \\
\hline Expected & $26 \cdot 4$ & $5 \cdot 4$ & 1 3.6 & I0. 6 & \\
\hline
\end{tabular}

(iv) $\mathcal{T}$ grandiflorum : 3 I clones, table 6

\begin{tabular}{|cc|c|c|c|c|c|}
\hline & $\mathrm{D}$ & 4 & 5 & 6 & $A b$ & $\mathrm{~T}$ \\
\hline $\mathrm{ab}$ & 4 & $2 \mathrm{I}$ & 2 & 3 & & \\
$\mathrm{aB}$ & 5 & & 5 & $\ldots$ & & \\
$\mathrm{AB}$ & 6 & & & $\ldots$ & & \\
\hline & & & & & \\
\hline Total & 47 & $\mathrm{I2}$ & 3 & $\ldots$ & 62 \\
\hline Expected & 44.7 & $\mathrm{I} 4.3$ & 0.72 & $2 \cdot 3$ & \\
\hline
\end{tabular}

Thus in $T$. cernuum and $T$. grandiflorum with parallel variation in $\mathrm{G}$ we have the situation in which we should expect that $\mathrm{I} \times 4 \rightleftharpoons 2 \times 5$. (In $T$. grandiflorum types 2 and 4 are also carried by other variants in respect of the other arm of the chromosome which can therefore be added to them.) The same situation can be used in $\mathrm{E}$ of $\mathcal{T}$. luteum where $\mathrm{E}_{2} \times 3 \rightleftharpoons \mathrm{E}_{\mathrm{I}} \times 4$. In each case we can represent the presence of an $\mathrm{H}$-segment by $A$ or $B$ and its absence by $a$ or $b$ (fig. 4 and table 14).

These recordings show that the higher frequencies of heterozygotes in $T$. luteum give rise, as we should expect, to more nearly random recombination. The lower frequencies in the other species give disturbed ratios indicating selective elimination of some types (table 14). Especially notable is the disappearance of the non-H 
type in $\mathcal{T}$. grandiflorum at the same time that other high- $\mathrm{H}$ types have appeared.

Contrasted with the situation in $T$. luteum is that in the three clones of Paris polyphylla (table 9). Here D and E chromosomes both vary exactly as in the $\mathrm{E}$ of $\mathcal{T}$. luteum giving the three types $\mathrm{ab}, \mathrm{aB}$ and $\mathrm{AB}$ and they vary completely in parallel; they are also all homozygous. Thus for the two pairs of chromosomes we have the formulæ :-
I. $\left\{\mathrm{D} \frac{\mathrm{ab}}{\mathrm{ab}}\right.$
$\left.E \frac{\mathrm{ab}}{\mathrm{ab}}\right\}$
2. $\left\{\mathrm{D} \frac{\mathrm{aB}}{\mathrm{aB}} \mathrm{E} \frac{\mathrm{aB}}{\mathrm{aB}}\right\}$
3. $\left\{\mathrm{D} \frac{\mathrm{AB}}{\overline{\mathrm{AB}}}\right.$
$\left.\mathrm{E} \frac{\mathrm{AB}}{\mathrm{AB}}\right\}$

This is the only example we have discovered of parallel variation between non-homologues within the complement. We therefore hope to obtain more specimens of this rare species.

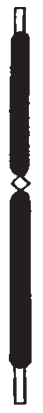

A

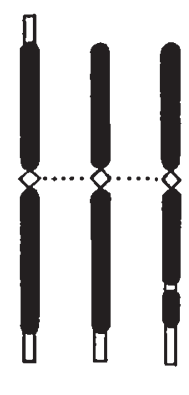

B1 $\quad B 2 \quad B 3$

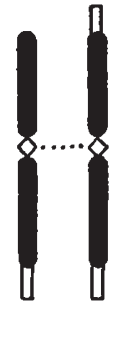

$\mathrm{Cl} C 2$
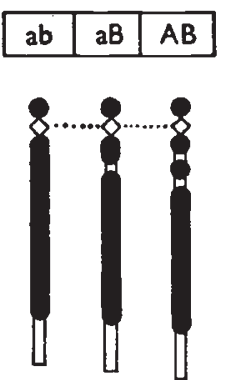

D1 D2 D3
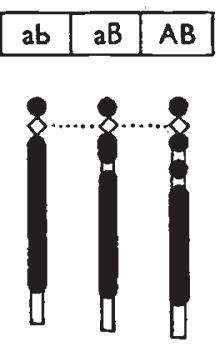

E1 E2 E3

Fig. 7.-Complement diagram for the three clones of Paris polyphylla. $\times$ c. 1200.

\section{EVOLUTION OF H-SEGMENTS}

Comparative. We now have consistent evidence from three genera of flowering plants, and from Triton (Callan, I942), of large chromosomes with blocks of heterochromatin capable of cold-starvation.

In each plant genus, Paris, Trillium and Fritillaria, there are species with and without heterochromatin. In each genus, also, the $\mathrm{H}$ segments have a characteristic pattern of distribution. In Fritillaria (of California) and Trillium (of Japan) it is proximal ; in Trillium (of America) proximal in some chromosomes, and distal in others; in Paris polyphylla it is chiefly distal. Further, the sizes of the Hsegments are characteristic of the species. Where the H-contents are lowest they lie in very short proximal segments as in Japanese Trillium and in Triton. Where they are highest they lie in long terminal segments.

Earlier studies have shown the lack of connection of $\mathrm{H}$-segments with crossing over in their distribution (Darlington, I936, I94I). In Trillium kamtschaticum $\mathrm{H}$-segments and crossing over are both proximally localised (Haga, I952). In Paris $\mathrm{H}$-segments are distal with crossing over proximal. And in Fritillaria $\mathrm{H}$-segments are proximal and crossing over unlocalised. 
The reason for this independence is no doubt that heterochromatin is condensed at the pachytene stage and from our evidence does not recombine by crossing over within its segments with euchromatin.

Where then do the $\mathrm{H}$-segments come from? The earlier view was based on a comparison between species and on the supposed relations of sex chromosomes in Drosophila. It was that $\mathrm{H}$-segments were allelic to euchromatin and the difference between the presence and absence of $\mathrm{H}$-segments in different species was due to a difference in genotypic control acting on the same genes (Darlington, I94I). But the comparison of variants within the plant species now shows that this transformation does not apply here (table io).

Both in Rutishauser's diagrams (1 956b, fig. 6) and in our measurements, the H-segments (especially in the B's of $\mathcal{T}$. luteum with terminal heterochromatin) appear to be additional when two homologues are compared. The simplest view is thus that they are replications of intercalated genes arising as a characteristic structural change in condensed

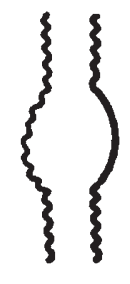

$\boldsymbol{a}$

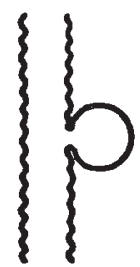

b

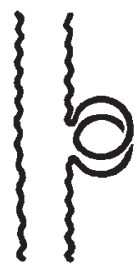

c

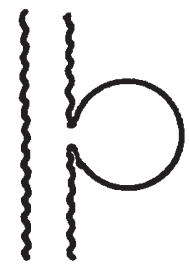

d

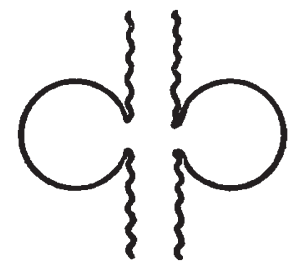

e

Fra. 8.-Pachytene diagram showing alternative relations of eu- and hetero-chromatin. (Wavy and straight lines) $a$, relations assumed in sex chromosomes of some animals, $b$-e, relations assumed in Trillium and other plants; $b$, original intercalation ; $c$, first duplication by illegitimate crossing over ; $d$, result of $c$ : heterozygote ; $e$, homozygote with non-pairing of heterochromatin.

segments (fig. 8). Such replications could preserve their individuality from the effects both of crossing over (which we know occurs in the adjacent euchromatin) and of other forms of breakage and reunion at pachytene. At pachytene H-segments in Fritillaria pudica seem to be condensed and incapable of pairing even when matched.

The evolutionary course with a replication of the $\mathrm{H}$-segments can be seen in all stages in Trillium and in each stage in some other group of flowering plants, as follows (fig. 9) :-

(i) H-segments arise as single units or genes. They become recognisable when they are replicated to form small blocks (as in Vicia faba). They are then often localised near the centromere (Trillium kamtschaticum).

(ii) Larger blocks are formed by further replication and give several blocks in each chromosome arm and in different chromosomes by translocation. The blocks are thus supernumerary, internally repetitive but freed from internal crossing over by pachytene condensation (Fritillaria pudica and T. stylosum). 
(iii) By crossing over these blocks are recombined. Some of the products of recombination, like $\mathrm{B}_{9}, \mathrm{~B}_{\mathrm{I}} \mathrm{I}_{1} \mathrm{~B}_{1} 3$ and $\mathrm{B}_{14}$, no longer occur in the same species. Their relics in different species show the consequences of crossing over and selective survival at an earlier stage of their history.

(iv) Where even larger blocks are required this is sometimes possible in a proximal position as in the California species of Fritillaria (La Cour, I95I). To produce this result intercalary H-segments can
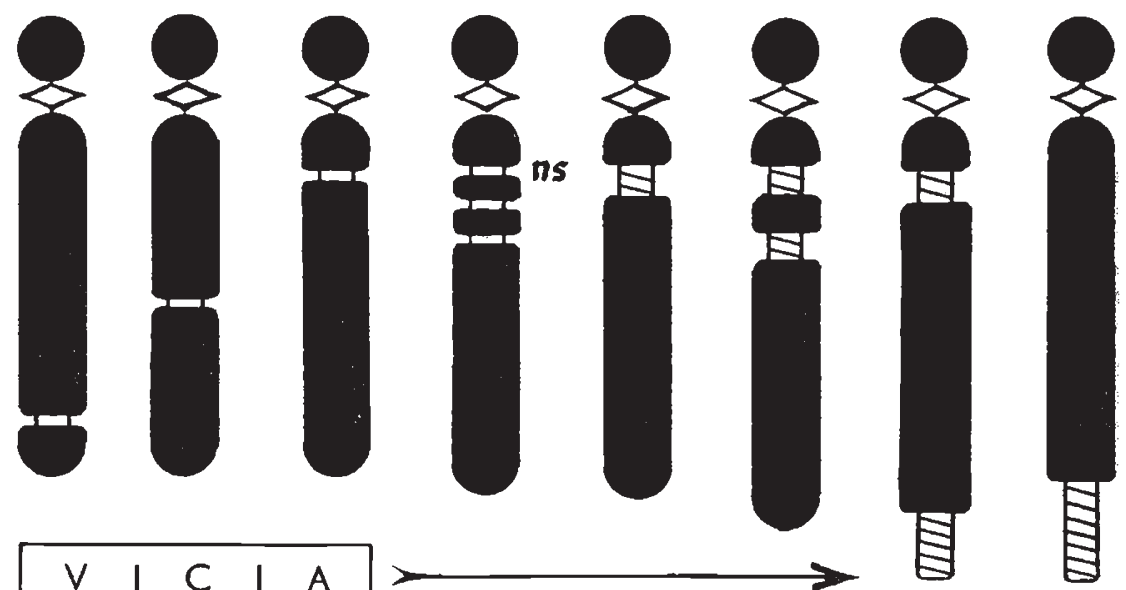

\section{\begin{tabular}{|lllll|}
\hline & I & C & I & $A$ \\
\hline
\end{tabular}}

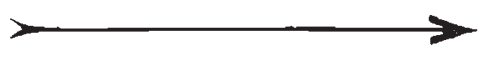

Japan : T R I L L I U M : America

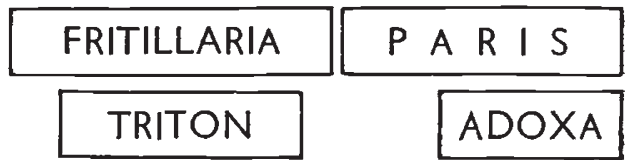

Fig. 9.-Eight types of $\mathrm{H}$-segment pattern found in single chromosome arms of six genera to show that evidence of an evolutionary sequence, represented by the arrow, may be derived from Trillium. ns, short segment capable of non-spiralising : only one per arm and only with the long arm distal to it.

Sources.-Vicia, McLeish, 1952 ; Trillium, Haga, 1956 ; Fritillaria, Darlington and La Cour, 1941 ; Paris, Darlington and La Cour, I938 ; Triton, Callan, 1942 ; Adoxa, Geitler, I940.

increase in size as shown by $\mathrm{C}_{3}$ in $T$. luteum and $\mathrm{D}_{5}{ }^{\prime}$ in $T$. ovatum; perhaps also in Lachenalia (Therman, I957).

(v) This faculty is not usually exploited perhaps because a longintercalary $\mathrm{H}$-segment is liable to obstructed division when starved. It will then stick at anaphase and so imperil the euchromatin distal to it (Darlington and La Cour, I940, Shaw, 1958). Translocation. towards the ends will thus usually be favoured. Hence will arise, first, the $\mathrm{BI}_{\mathrm{I}}$ or $\mathrm{B}_{\mathrm{I}} 2$ pattern, and later the terminal position which is established in B and D in most American species of Trillium and is beginning in $\mathrm{C}$. Owing to their liability when starved to form bridges. which break unequally these end segments can be rapidly extended. The extensions will again always be repetitive. 
(vi) Thus the species of Trillium ( $T$. chloropetalum and $T$. recurvatum) with the highest $\mathrm{H}$-content represent the nearly final stage in this evolution. Similarly the terminal position for the $\mathrm{H}$-segments has almost displaced the intercalary in Paris polyphylla and altogether displaced it in Adoxa moschatellina (Geitler, 1940). This last stage is compatible with a very high $\mathrm{H}$-content. It is therefore most prominent and was the first to be observed.

In the evolution of Trillium, as we see, $\mathrm{H}$-segments seem to jump from proximal to distal positions. Why? In species growing where temperatures are likely to lead to DNA starvation the mechanical consequences of this starvation will no doubt affect the distribution of $\mathrm{H}$-segments. The activities of the $\mathrm{H}$-segments in the resting nucleus, where (by contrast with nucleoli) they seem to need contact with the nuclear membrane, may however also affect their distribution ( $c f$. Therman, 1957). Probably for this reason H-segments so rarely lie in the middle of long arms or at the end of short arms.

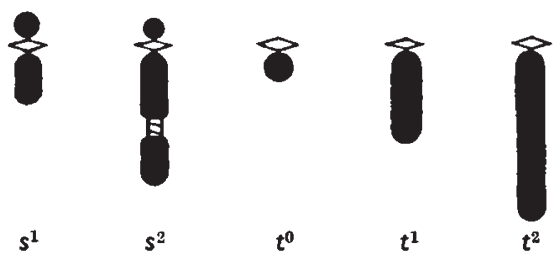

Fig. 10.-The five types of supernumerary found in Trillium and Paris with their symbols : sub-telocentric, $s$; telocentric, $t . \quad s^{1}-2 \cdot 5 \mu, s^{2}-9 \mu, t^{0}-1 \cdot 5 \mu, t^{1}-4 \cdot 0 \mu, t^{2}-10 \mu . \quad \times c .1800$.

\section{SUPERNUMERARY CHROMOSOMES}

In addition to the five pairs of what we may call autosomes, sixty-one plants had supernumerary chromosomes. These we refer to as $f$ rather than $\mathbf{B}$ in Trillium. They have not been found in the Japanese species.

Constancy. In tables 2-9 the total numbers of plants in each clone are given, the clones being defined by the uniformity of $\mathrm{H}$-segment pattern in the several plants of each. Within rhizomes (even four branching rhizomes of $\mathcal{T}$. erectum) the numbers of supernumeraries have proved to be constant. Evidently, therefore, somatic change, if it occurs, is less frequent than elsewhere (cf. Håkansson, 1956, on Godetia ; Bosemark, I957, on Poa trivialis).

In those clones which include several plants, however, the plants may vary in number of supernumeraries. Thus, of our I 38 " clones", forty-seven had supernumeraries. Of these, seventeen, or nearly all of those which included several plants, varied in the number of supernumeraries. We shall refer to the groups with constant supernumeraries as sub-clones.

How do these sub-clones arise? In T. erectum, T. sessile, T. grandiflorum and $P$. polyphylla there are altogether ten clones with none and 
with one supernumerary. In these cases we believe we may be concerned with genuine sub-clones derived by loss of the supernumerary at mitosis. There are, however, discontinuous types of sub-clone in $T$. erectum (clones I I, I4 and 23 ) and $T$. sessile (clone $I$ ). In the extreme case two sub-clones had none and six fragments. Such subclones must be really different clones from different seedlings.

\section{TABLE I 5}

Numbers of clones with different numbers of supernumeraries in Trillium and Paris ( 128 clones, $35 \mathrm{~s}, 35 \mathrm{t}$ )

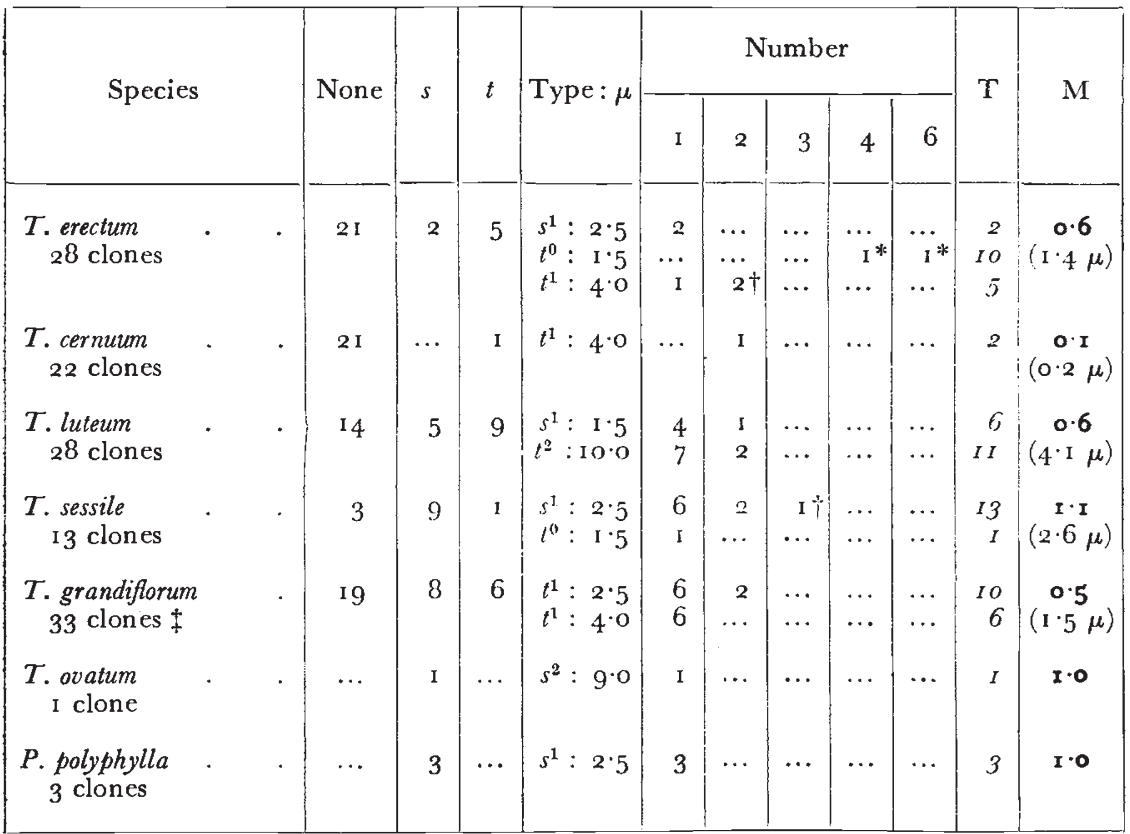

* Sub-clones had no ff.

$\dagger$ Sub-clones had $\mathrm{I}$ and off.

$\ddagger$ Three sub-clones in terms of autosomes were of the three different supernumerary types and are counted as 3 clones here.

Types. The supernumeraries of Trillium and Paris are of two main types, sub-telocentric $(s)$ and telocentrics $(t)$. There is never more than one type within an individual and there are no iso-chromosomes. The centromeres are thus fairly stable and both types may be in their original form, $s$ being derived by breakage of heterochromatin and $t$ by misdivision of the centromere (table I $_{5}$ ).

All the supernumeraries save one in $\mathcal{T}$. ovatum are entirely euchromatic. There are five sizes of supernumerary from $15 \mu$ to $10 \mu$ in length. The ro $\mu$ and $4 \mu$ types never exceed two in number; the $2.5 \mu$ type three, and only the $I .5 \mu$ type reaches six. Indeed they represent a source of variation, reaching Io per cent. in $T$. luteum, of the length of euchromatin which, otherwise, within the autosomes, is almost invariable. As a fraction of the whole complement this 
corresponds to the variation in heterochromatin afforded by the H-segments.

The supernumeraries in grasses (Zea, Secale, Sorghum, Anthoxanthum, Festuca and Poa) are heterochromatic or largely so ( $c f$. Bosemark, 1957). In these grasses, however, there is little heterochromatin in the autosomes. In maize, indeed, heterochromatin seems to have been developed in the autosomes of improved varieties to replace what has been lost in the supernumeraries (Darlington and Upcott, I94I).

Boosting Mechanisms. In plants we know four mechanisms which conduce to the maintenance or increase of supernumeraries and compensating for their loss of meiosis.

(i) Pairing and spiralisation of supernumeraries lapses at first metaphase of meiosis and the univalents pass to one pole (Parthenium : Catcheside, I950).

(ii) Both chromatids of supernumeraries pass to the generative nucleus at the first pollen mitosis (Sorghum, Secale, Anthoxanthum, Festuca, Phleum, etc.: Darlington and Thomas, 1941, Müntzing, I 946$)$.

(iii) Pollen tubes with supernumeraries grow faster than those without them: hence they preferentially fertilise the eggs (Zea, Roman, I948).

(iv) Supernumeraries arepre ferentially included at embryosac meiosis in the egg-cell nucleus (Lilium, Kayano, I956; Trillium, Rutishauser, I956a).

In Trillium the supernumeraries are normally distributed in the pollen mother cells with some elimination (Sparrow, I952). In the embryo-sac they are boosted. It is significant that only heterochromatic supernumeraries can be directed in the pollen grain mitosis since the direction depends on sticking of $\mathrm{H}$-segments at anaphase. Euchromatic supernumeraries depend on embryo-sac direction.

Evidently supernumeraries are keeping themselves going in populations of nearly all the American species of Trillium. The boosting mechanism compensates for loss at meiosis. Plants with and without supernumeraries are competing in nature on equal terms, as they are in so many hundreds of other plant genera consisting of herbaceous or annual diploid species.

Like all other forms of balanced polymorphism each alternative is advantageous in a part of the population.

\section{ORIGINS OF POLYMORPHISM}

(i) Levels and modes

Balanced polymorphism in the sense in which Ford (1957) has developed the notion depends on the maintenance by selection of an equilibrium between alternative or mutually replaceable genotypes in an interbreeding population. It differs from new or sporadic variation in that the two alternatives will have had a long time to 
modify their own genetic neighbourhood by mutual selection, a process which will always encourage the development of a super-gene. Any development of this kind of discontinuity is an integrative and evolutionary process like that of species formation but on a reduced scale. Its study may rely on the phenotype, as when it concerns mimicry, caste-formation, blood antigens, breeding systems and cytoplasmic polymorphisms. Or it may rely on the chromosomes as when it concerns inversions, interchanges, fragmentation, supernumerary chromosomes, iso-chromosomes and H-segments. Or again, it may combine both methods as we see in sex determination, the manytiered sex-ratio system of Drosophila, or the complex-heterozygote in Enothera.

The combination of the chromosome and phenotypic approaches to polymorphism shows us an interlocked system of interpretation whose parts need to be disentangled. Crossing over suppression and floating structural variation are opposite aspects of the genetic isolation of segments and the evolution of super-genes ( $c f$. Darlington, I956). The combination of the two methods and aspects illuminates the immense range of studies of polymorphism, by analogy, by antithesis and even by paradox.

Take first an analogy. All methods demonstrating parallel polymorphism are also means of tracing phylogeny. Thus with the hybrid species of Enothera the variable interchanges show parallel polymorphism, while the constant and conservative pairing segments preserve the phylogeny throughout whole sections of the genus. Similarly, the sex-ratio inversion complex in Drosophila and the bloodgroup genes in men and monkeys demonstrate parallel polymorphism and reveal phylogeny over long ranges of descent. Parallel polymorphism in the $\mathrm{H}$-segments of Trillium, therefore, although unusually striking, merely extends a principle already revealed in several other ways.

Secondly, consider an antithesis. New variations arising in the chromosomes, whether mutations or structural changes, encounter various kinds of situation in which they can spread. These situations may differ externally (Mather, I955). They may also differ internally. On the one hand, there is that in which they benefit by genetic isolation from their alternatives at the diploid level. Such variations underlie the separation of species. On the other hand, there is that in which they benefit by genetic combination with their alternatives at the diploid level. This situation favours genetic isolation at the haploid level. It underlies polymorphism; it underlies the development of supergenes and gene complexes; and it underlies developments of $\mathrm{H}$-segments and supernumeraries such as we have been considering.

Again, within this second group, we have an antithesis between those in which selection is indifferent as between heterozygote and homozygote and those in which it favours the heterozygote. Now polymorphisms which depend on the advantage of the heterozygote 
may lead to the complex heterozygote in which no homozygotes exist (as first discussed by Bateson, 1902). The population of zygotes has then ceased to be polymorphic but the polymorphism may be said to have been transposed to the haploid level. This happens with both interchange and fragmentation polymorphisms, and with both together in Campanula ( $c f$. Darlington, 1956a and $b$ ).

Comparison of these situations indicates that selection maintains. or advances a polymorphism either by disfavouring certain homozygotes inherently or by disfavouring them only when they are excessively frequent, that is by encouraging differences as such. $\mathbf{H}$ segments and supernumeraries prosper, as we have seen, by the second means : not by favouring heterozygotes, but by generating

TABLE 16

Contrasted properties of the two replicatory types of polymorphism

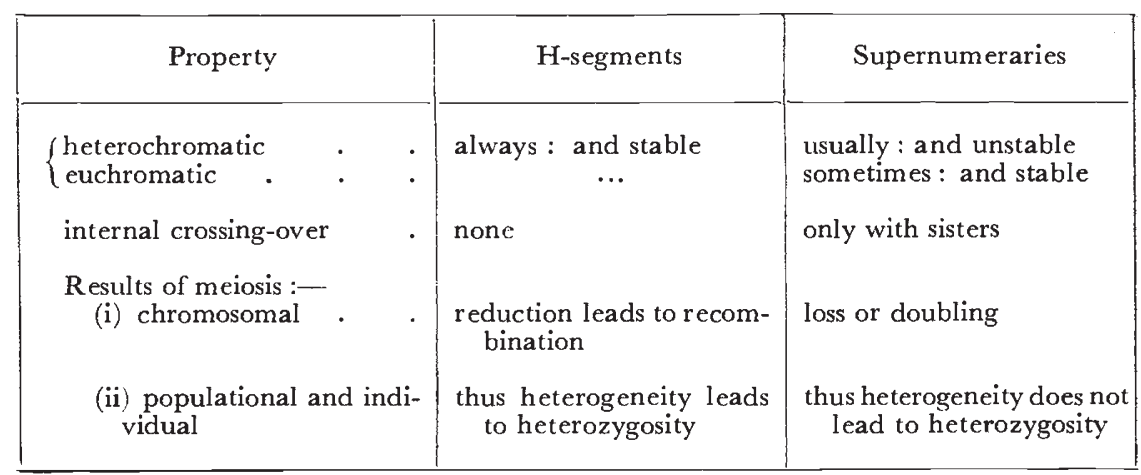

diversity. They both provide a source of variation for the population : they pre-adapt it to the needs of a fluctuating environment. Although both of them are based on replication they achieve this end by contrasted means (table I6).

\section{(ii) Alternative types}

The contrast of properties between H-segments and supernumerary chromosomes is instructive in two respects. First, it shows that heterochromatin is always relatively unstable when it makes a whole chromosome: it is liable to misdivision and also fragmentation. But it is relatively stable when wedged between large segments of euchromatin. Secondly, it shows us that supernumeraries can be either heterochromatic or euchromatic.

Now, the established supernumeraries of Trillium and Paris are euchromatic. Are they then co-operating with the heterochromatic material in the autosomes? Or are the two in some sense competing? Can it be that the $\mathrm{H}$-segments in providing variation within the autosomes in respect of heterochromatin have freed the supernumerary mechanism as a basis of variation in euchromatin?

Answers to these questions can be obtained by comparing the occurrence of supernumeraries in species with different H-contents, 
that is in diploid species, for established polyploids have neither. There is evidently a correlation (table I 7). This correlation should apply to other species of Trillium. T. ovatum, recurvatum and chloropetalum should also prove to have euchromatic supernumeraries when large samples are studied.

This comparison makes it probable that the two sources of variation in heterochromatin and in euchromatin are co-operating and not competing in Trillium. It is not to be supposed that this is a general principle. Nor is it to be supposed that it answers the basic questions. We are inclined to suppose that the $\mathrm{H}$-segments of Trillium, on the one

TABLE 17

Relation between presence of heterochromatin in autosomes and euchromatic supernumeraries in diploid species of Trillium and Paris

\begin{tabular}{|c|c|c|c|}
\hline \multirow{4}{*}{ 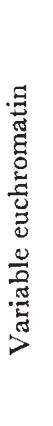 } & & \multicolumn{2}{|c|}{ Variable heterochromatin } \\
\hline & & Much: $>5$ per cent. & Little $:<5$ per cent. \\
\hline & With supernumeraries & $\begin{array}{l}\text { Trillium: } \\
5 \text { American species } \\
\text { Paris polyphylla }\end{array}$ & none \\
\hline & Without supernumeraries & none & $\begin{array}{l}\text { Trillium: } \\
3 \text { Japanese species } \\
T . \text { stylosum }\end{array}$ \\
\hline
\end{tabular}

hand, are replications of the same stuff and of great age. The supernumeraries, on the other hand, being euchromatic, are not replicated but are differentiated internally. In view of the great heterogeneity of $T$. erectum which had three types of supernumerary in twentyeight clones, each no doubt qualitatively different from the others, we must also assume that they are constantly being replaced.

The great development of heterochromatin in Trillium is an indication of mechanically or physiologically unusual conditions. Its situation is nevertheless important as showing how different groups of organisms with similar mechanisms of heredity may have entirely different mechanisms of variation.

It shows us particularly that, at the chromosome level, we may contrast two kinds of polymorphism. Those which are phenotypically recognisable are allelic. Those which are not so recognisable are replicatory.* Pairing in haploids shows that a great deal of replicatory variation is going on under the surface in most organisms. But supernumerary chromosomes and $\mathrm{H}$-segments are visibly replicatory. They both arise as replications and they both seem to proliferate by further replication. Their action is non-specific and incapable of

* It would be far-fetched to call them Morganian and Batesonian, but we may note that replication implies presence-and-absence. 
heterosis. This we expect of heterochromatin. We may also expect it of any other replication beyond the diploid level such as that of supernumeraries.

The suggestion that the replicatory polymorphisms act merely by increasing variability in a quantitative sense presents us with an interesting contradiction. For such replicatory variation would seem. likely to provide the basis of certain kinds of polygenic variation. Now polygenic variation has not been thought of as connected with polymorphism because its discontinuity is invisible except at the chromosome level. But so also is the contrast between the allelic and the replicatory situations.

The present studies continue a development which has proceeded unnoticed ever since the rediscovery of Mendelism. For throughout this time we have been increasingly impressed with the effect of chromosomes as determining heredity. This proper interest in determining heredity has distracted us, however, from their other property of concealing variation. Or, as we may put it, their property of carrying invisible but useful ballast.

Most new alleles, being recessives, most structural changes in chromosomes, most polygenic variation, most heterochromatin, most supernumerary chromosomes, and even a great deal of polyploidy : all these are concealed from us. Each is a cryptic variation. All of them combined give us cryptic species. Heredity we discover is a reservoir of concealed variation. And it is in this respect that genetics has most changed our view of the process of evolution from that which could be envisaged in the nineteenth century by Lamarck or by Darwin or indeed by anyone except Mendel.

\section{SUMMARY}

I. The heterochromatin in H-segments has been mapped by DNA-starvation of the chromosomes in samples of ten species of Trillium and one of Paris (table I, figs. I-8).

2. There are three degrees of starvation depending on differences in physiological conditions ( $c f$. Shaw, 1958, 1959, Heyes and Shaw, I 958).

3. In the five pairs of autosomes (A-E) the 273 plants showed 142 types of chromosome complement differing in number and position of $\mathrm{H}$-segments. Some complements found in several plants are assumed to be clonal replicates for the present purpose (table io).

4. The proportion of heterochromatin varies in the group from 0.5 per cent. to 25 per cent. and over a range of $I: 4$ within species (table I I).

5. Of the ten supposed species of Trillium two pairs overlap in external form and in $\mathrm{H}$-segment variation. There are thus eight genetic species. Of these, four in large samples from North Carolina showed wide internal variation. 
6. Size, position and frequency relations between $\mathrm{H}$-segments revealed five principles :-

(i) Homologous chromosomes within species vary in their $\mathrm{H}$-segments mainly owing to addition to the euchromatin and not to conversion of it.

(ii) Homologous chromosomes vary in parallel within and between species. This indicates a parallel balanced polymorphism derived from common ancestors.

(iii) Whole complement comparisons indicate that the $\mathrm{H}$-segments began in Trillium in intercalary positions near the centromere, the Fritillaria-Vicia pattern. Later they have been shifted to the ends to give the Paris pattern in one or two chromosomes.

(iv) Allelic relations show that heterozygosity is not favoured (table i 3 ).

(v) Double-allelic relations show that crossing over is free in $\mathcal{T}$. erectum if not in $P$. polyphylla (fig. Io, table I4).

7. In sixty-one plants of the seven species having most heterochromatin there were supernumerary chromosomes. All were of small standard types, subtelo- or telo-centric, and all, save one, were entirely euchromatic.

8. The correlation between supernumerary chromosomes and Hsegments is not unexpected. They provide corresponding sources of variability in euchromatin and in heterochromatin. Both are most strongly developed in diploid species. Both afford a replicatory and non-specific, as opposed to an allelic and specific basis of polymorphism.

\section{REFERENCES}

BAILEY, L. H. I94I. The Standard Cyclopedia of Horticulture. Macmillan, New York. Bailey, P. C. 1949 . Differential chromosome segments in Trillium erectum L. Bull. Torrey Bot. Club, 76, 3r9-336.

BAILEy, P. G. 1952. Differential reactivity in six species of Trillium. Bull. Torrey Bot. Club, 79, $45^{\mathrm{I}-45^{8}}$.

BAILEY, P. C. I 954 . Differential reactivity of chromosomes as an indicator of species in Trillium. Bot. Gaz., $115,24 \mathrm{I}-248$.

Bateson, w. rgo2. The facts of heredity, etc. Sci. Papers (Cambridge, r928), 2, 37 .

воOтHROYD, E. R. 1953. The reaction of Trillium pollen tube chromosomes to cold treatment during mitosis. F. Heredity, 44, 3-9.

BOSEMARK, N. o. 1957. Further studies on accessory chromosomes in grasses. Hereditas, 45, 236-297.

BRItTON AND BROWN (AND GLEASON, H. A.). 1952. Flora of the United States, etc. New York.

Gallan, H. G. 1942. Heterochromatin in Triton. P.R.S., B, 130, 324-335.

CATCheside, D. G. 1950. The B-chromosomes of Parthenium argentatum. Genet. Iber., 2, 139-1 48 .

Darlington, G. D. 1936 . The external mechanics of the chromosomes. P.R.S., B, I2I, 264-3I 9 . 
DARlington, C. D. I94I. Polyploidy, crossing over and heterochromatin in Pairs. Ann. Bot., 5, 203-2 I 6.

DARLington, c. D. I956a. Natural populations and the breakdown of classical genetics. P.R.S., B, I45, 350-364.

Darlington, C. D. I 9566 . Chromosome Botany. Allen \& Unwin, London.

DARLington, C. D. I957. Messages and movements in the cell. Conference on Chromosomes. Willink, Zwolle, Netherlands.

DARlington, C. D., AND LA COUR, L. F. I938. Differential reactivity of the chromosomes. Ann. Bot., 2, 615-625.

DARLINGTON, C. D., AND LA COUR, L. F. I 940 . Nucleic acid starvation of chromosomes in Trillium. 7. Genet., 40, I85-213.

Darlington, C. D., AND LA COUR, L. F. I94I. The detection of inert genes. $\mathcal{F}$. Heredity, 32, I I 5-I 2 I.

DARlington, C. D., AND LA cour, L. F. 1945. Chromosome breakage and the nucleic acid cycle. F. Genet., 46 , 108-267.

DARLington, C. D., AND LA cour, L. F. 1950. Hybridity selection in Campanula. 7. Heredity, 4, 21 7-248.

DARLINGTON, C. D., AND THOMAS, P. T. 194I. Morbid mitosis and the activity of inert chromosomes in Sorghum. P.R.S., B, I30, I 22-I 50.

DARLington, C. D., AND UPCOTT, M. B. 194I. The activity of inert chromosomes in Zea mays. F. Genet., $4^{I}, 275-296$.

FORD, C. E., HAMERTON, J. L., AND SHARMAN, G. B. 1957. Chromosome polymorphism in the common shrew. Nature, I80, 392-393.

FORD, E. B. 1957. Polymorphism in plants, animals and man. Nature, 180, I3! 5-I3I9.

GeItLeR, L. 1940. Temperaturbedingte Ausbildung von Spezialsegmenten au Chromosomsnenden. Chromosoma, I, 554-56I.

Giles, R. A., AND wilson, G. B. I956. A cytological and morphological study of two populations of Trillium grandiflorum. Cytologia, 2I, 376-383.

GRAY (AND FERNALD, M. L.). 1950. Manual of Botany, New York.

HAGA, т. 1952. Failure of pairing in Trillium kamtschaticum. Fap. F. Genet., 27, 72-74.

HAGA, T. I 956 . Genome and polyploidy in the genus Trillium. VI. Hybridisation and speciation by chromosome doubling in nature. Heredity 10 , 85-98.

haGa, T., AND KURABAyAShI, M. 1953. Genome and polyploidy in the genus Trillium. VI. Differential reaction of chromosome segments to low temperature. Cytologia, ${ }_{1} 8$, I $3-28$.

haGa, T., AND KURABAYAShi, M. I954. Genome and polyploidy in the genus Trillium. V. Natural populations of kamtschaticum. Mem. Fac. Sci. Kyushu Univ. (E) Biol., I, I59-185.

HÅKANSSON, A. I956. Spontaneous chromosome variation in the roots of a species hybrid. Hereditas, 32, 39-59.

HEYES, J. K., AND SHAW, H. W. 1958. Chemical determination of Deoxyribonucleic acid in root tips before and after cold treatment. Nature I8I, I377-1 388.

KAyano, H. I956. Cytogenetic studies in Lilium callosum. II. Preferential segregation of supernumerary chromosomes. Mem. Fac. Sci. Kyushu Univ. (E) Biol., 2, 53-60.

KURABAYASHI, M. 1957. Evolution and variation in Trillium. IV. Natural populations of $\mathcal{T}$. kamtschaticum. Fap. F. Bot., $16, \mathrm{I}-45$.

LA COUR, L. F. 195I. Heterochromatin and the organisation of nucleoli in plants. Heredity, 5, 37-50.

MGLeIsH, J. 1952. The action of maleic hydrazide in Vicia. Heredity, 6 (Sup. Chr. Breakage), 125-147.

MATHeR, K. I955. Polymorphism as an outcome of disruptive selection. Evolution, $9,52-6$ I. 
MÜNTZING, A. 1946. Cytological studies of extra fragment chromosomes in rye.

III. The mechanism of non-disjunction at pollen mitosis. Hereditas, 32, 97-I I9. Roman, N. 1948. Directed fertilisation in maize. P.N.A.S., 34, 36-42.

RUTISHAUSER, A. 1956a. Genetics of fragment chromosomes in T. grandiflorum. Heredity, Io, I95-204.

RUTishauser, A. I 9566 . Chromosome distribution and spontaneous chromosome breakage in T. grandiflorum. Heredity, ro, 376-407.

RUTISHAUSER, A., AND LA COUR, L. F. 1956. Spontaneous chromosome breakage in hybrid endosperms. Chromosoma, 8, 3 1 7-340.

SHAW, G. W. I950. A rapid permanent method for chromosomes. Nature, ${ }_{165}, 408$. sHAw, G. W. I956. The preparation of chromosomes. Microscope, II, 43-45.

sHAw, G. w. 1958. Adhesion loci in the differentiated heterochromatin of Trillium species. Chromosoma $9 ;$ 292-304.

sHAw, G. W. 1959. The nature of differential reactivity in the heterochromatin of Trillium and Paris species. Cytologia (in the press).

SPARROW, A. H., et al. 1952. Distribution and behaviour of supernumerary chromosomes during microsporogenesis in a population of T. erectum L. Amer. Nat., $86,277-292$.

THERman, E. 1957. Chromocentres in the mitosis of Lachenalia. Arch. Soc. Vanamo, II, I 89-193.

wallace, B. 1948. Studies on "Sex-Ratio" in Drosophila pseudoobscura. I. Selection and "Sex-Ratio". Evolution, 3, 189-2 I 7.

WILSON, G. B., AND воOTHROYD, E. R. I94I. Studies in differential reactivity I. The rate and degree of differentiation in somatic chromosomes. Canad. F. Res., I9, 400-412.

WILSON, G. B., AND воотHRoYD, E. R. 1944. Temperature-induced differential contraction in the somatic chromosomes of Trillium erectum. Canad. F. Res., 22, I05-II 9 . 